\title{
Constraining compactness and magnetic field geometry of X-ray pulsars from the statistics of their pulse profiles
}

\author{
M. Annala and J. Poutanen
}

Astronomy Division, Department of Physics, PO Box 3000, 90014 University of Oulu, Finland

e-mail: [marja.annala; juri.poutanen]@oulu.fi

Received 26 June 2009 / Accepted 5 August 2010

\section{ABSTRACT}

\begin{abstract}
Context. The light curves observed from X-ray pulsars and magnetars reflect the radiation emission pattern, the geometry of the magnetic field, and the neutron star compactness.

Aims. We study the statistics of X-ray pulse profiles in order to constrain the neutron star compactness and the magnetic field geometry. Methods. We collect the data for 124 X-ray pulsars, which are mainly in high-mass X-ray binary systems, and classify their pulse profiles according to the number of observed peaks seen during one spin period, dividing them into two classes, single- and doublepeaked. We find that the pulsars are distributed about equally between both groups. We also compute the probabilities predicted by the theoretical models of two antipodal point-like spots that emit radiation according to the pencil-like emission patterns. These are then compared to the observed fraction of pulsars in the two classes.

Results. Assuming a blackbody emission pattern, it is possible to constrain the neutron star compactness if the magnetic dipole has arbitrary inclinations to the pulsar rotational axis. More realistic pencil-beam patterns predict that $79 \%$ of the pulsars are doublepeaked independently of their compactness. The theoretical predictions can be made consistent with the data if the magnetic dipole inclination to the rotational axis has an upper limit of $40^{\circ} \pm 4^{\circ}$. We also discuss the effect of limited sensitivity of the X-ray instruments to detect weak pulses, which lowers the number of detected double-peaked profiles and makes the theoretical predictions to be consistent with the data even if the magnetic dipole does have random inclinations. This shows that the statistics of pulse profiles does not allow us to constrain the neutron star compactness. In contrast to the previous claims by Bulik et al. (2003, A\&A, 404, 1023), the data also do not require the magnetic inclination to be confined in a narrow interval.
\end{abstract}

Key words. methods: statistical - pulsars: general - stars: neutron - X-rays: binaries

\section{Introduction}

X-ray pulsars have been discovered in the 1970s (Giacconi et al. 1971) and have served as laboratories to study the neutron star (NS) physics since then. Most of them are members of binary systems and accrete matter through wind or via disk from a highmass companion. Because of a large magnetic field strength (typically $10^{12} \mathrm{G}$ ) the material is channeled onto small spots at the magnetic poles. Here the relativistically moving plasma is decelerated in a radiative shock near the surface and this subsonically settling plasma radiates in the X-ray band (see e.g. Davidson \& Ostriker 1973; Basko \& Sunyaev 1976). Pulsations are observed if the magnetic field is inclined relative to the rotation axis. Even stronger field pulsars (magnetars) have been discovered recently, which operate by dissipating magnetic energy (see e.g. review by Mereghetti 2008). Studies of the pulse profiles of individual pulsars allow one to constrain the emission pattern of the hotspots (or accretion columns) at the NS surface as well as the geometry of the magnetic field (see e.g. Yahel 1980; Bulik et al. 1995; Kraus et al. 1996).

The number of known pulsars in the Milky Way and the nearby Small and Large Magellanic clouds is already above a hundred. The quality of the data is also improving because of the sensitive X-ray/gamma-ray observatories such as $R X T E$ and INTEGRAL and the long observing times. The first pulsars have already been discovered in M 31 (Trudolyubov et al. 2005) and in even more distant galaxies (Trudolyubov et al. 2007; Trudolyubov 2008). A large number of pulsars allows us to use a statistical approach to constrain the NS parameters. Because of the gravitational light bending, the more compact the star, the larger the fraction of the NS surface that is visible to an observer at all times. This increases the probability to observe two radiative poles in one rotational period and affects the relative fraction of the single- and double-peaked pulse profiles. The second important parameter that affects the pulse profile is the positions of the hotspots relative to the rotational axis (which are defined in the simplest case by the inclination of the magnetic dipole).

For any reasonable NS parameters one expects that both poles are visible in a large majority of pulsars. However, the number of observed single- and double-peaked profiles is not so different. This was already noticed by Wang \& Welter (1981) and later by Bulik et al. (2003, B03 hereafter). This discrepancy can be explained if the inclination between the magnetic dipole and rotational axis is not randomly distributed, but if there is a strong bias towards alignment. In the present study, we consider a sample of 124 pulsars with better quality data than were available before. We study in detail the statistics of double-peak profiles for various pencil-like emission pattern. We also discuss the effect of the detection threshold that can significantly affect the observed fraction. We then derive useful analytical formulae that describe the probabilities of observing certain types of pulsars. And finally we compare our theoretical model to the data.

\section{Data selection and classification of light curves}

The light curves of X-ray pulsars can be classified according to the number of pulses per period. The observed pulse profiles tend 
to simplify with increasing energy, and the multiple-peaked profiles change into double- or single-peaked (see e.g. Nagase 1989; Bildsten et al. 1997). In order to reduce possible effects of the photoelectric absorption and the cyclotron lines, the pulse classification is done at the highest possible energies (typically above $10 \mathrm{keV})$. We use the published light curves of X-ray pulsars from several sources. Therefore the data are inhomogeneous. A significant number of light curves are produced in an nonappropriate energy range, which makes it difficult to conclude anything about the number of poles visible to the observer and details of the beam shape.

Bulik et al. (2003) have studied the profiles of 88 pulsars (not 89 because one of the sources, 1WGA J1958.2+3232, turned out to be an intermediate polar, Negueruela et al. 2000), which have been divided into three different groups. The first group consisted of 46 pulsars, which were easy to classify. The second group consisted of 31 pulsars, which were difficult to classify and the third group had 11 pulsars for which there were no good quality light curves available in 2003. We have scanned the latest literature for the light curves from the same sources and also added to our sample all newly discovered X-ray pulsars. All together the sample now consists of $124 \mathrm{X}$-ray pulsars. The pulsars are placed in two different categories depending on the kind of the data: those which have the profiles observed above $10 \mathrm{keV}$ and those that do not. The first category contains 60 pulsars and the second one 64 pulsars. Their data are given in Tables 1 and 2, respectively, together with their classification (i.e. the number of pulses observed). A similar classification from B03 (who found 38 double-peaked light curves out of 88 sources) is also shown for comparison. Only the pulsars residing in high-mass X-ray binaries and Be-transients as well as magnetars are included, while all disk-accreting systems residing in low-mass X-ray binaries are excluded. This is because the disk can seriously affect the light propagation from the secondary pole to the observer (e.g. Ibragimov \& Poutanen 2009).

A couple of the classified sources (e.g. 4U 1538-52) show profile changes in the energy ranges above $10 \mathrm{keV}$, and the secondary pulse was not observed in every energy band. The most probable reason for this is the cyclotron absorption. These sources were classified as double-peaked. In two sources, GX 304-1 and AX J1841.0-0536, pulsations become weak as the energy increased. These sources were classified as singlepeaked.

In general, the probability of observing $M$ double-peaked pulsars out of $N$ sources, is given by the binomial distribution. Because our data set is very large and the observed number of pulsars of both types is similar, we can use the normal distribution instead. The estimation of the probability is $p=M / N$ and its error is $\sqrt{p(1-p) / N}$. In our classification of all pulsars in Tables 1 and 2 we found 55 double-peaked light curves out of 124 sources, which gives the probability of observing doublepeaked profiles

$p_{0}=0.44 \pm 0.04$.

For those pulsars which have light curves above $10 \mathrm{keV}$ (Table 1), we have 33 double-peaked pulsars out of 60 sources, which gives the corresponding probability of

$$
p_{1}=0.55 \pm 0.06 \text {. }
$$

This is still consistent with $p_{0}$ within $2 \sigma$. Excluding the magnetars from the list of pulsars would change the probabilities very little to $p_{0}^{\prime}=0.43 \pm 0.05$ and $p_{1}^{\prime}=0.52 \pm 0.07$.
Table 1. Light curve classification of $60 \mathrm{X}$-ray pulsars observed at energies above $10 \mathrm{keV}$.

\begin{tabular}{|c|c|c|c|c|}
\hline Name & $P_{\text {spin }}{ }^{a}$ & Pulses $^{b}$ & $\mathrm{~B} 03^{c}$ & Ref. \\
\hline RX J0051.8-7310 & 16.6 & 1 & & 1 \\
\hline RX J0052.1-7319 & 15.4 & 2 & 2 & 2 \\
\hline XTE-SMC95 & 95 & 1 & & 3 \\
\hline SMC X-2 & 2.37 & 1 & 1 & 4 \\
\hline XTE J0055-724 & 59 & 1 & 1 & 5 \\
\hline SMC X-1 & 0.71 & 2 & 2 & 6 \\
\hline RX J0117.6-7330 & 22 & 2 & 2 & 7 \\
\hline 2S $0114+650$ & 9828 & 1 & 1 & 8 \\
\hline 4U $0115+63$ & 3.6 & 1 & 1 & 9,16 \\
\hline $4 \mathrm{U} 0142+614^{d}$ & 8.7 & 2 & 2 & 10 \\
\hline RX J0146.9+6121 & 1408 & 1 & 1 & 11 \\
\hline V $0332+53$ & 4.37 & 2 & 1 & 12 \\
\hline 4U $0352+309=X$ Per & 835 & 1 & 1 & 13 \\
\hline EXO 053109-6609.2 & 13.7 & 2 & & 14 \\
\hline LMC X-4 & 13.5 & 1 & 1 & 15 \\
\hline $1 \mathrm{~A} 0535+26$ & 105 & 2 & 2 & 16 \\
\hline MXB 0656-072 & 160 & 1 & & 17 \\
\hline 4U 0728-25 & 103 & 2 & 2 & 18 \\
\hline RX J0812.4-3114 & 31.9 & 2 & 2 & 19 \\
\hline GS 0834-430 & 12.3 & 2 & 2 & 16,20 \\
\hline Vela X-1 & 283 & 2 & 2 & 16,21 \\
\hline GRO J1008-57 & 93.5 & 1 & 1 & 16 \\
\hline 1A $1118-616$ & 405 & 1 & 1 & 16,22 \\
\hline Cen X-3 & 4.82 & 2 & 2 & 16,23 \\
\hline 1E 1145.1-6141 & 297 & 2 & 1 & 16,21 \\
\hline 4U 1145-619 & 292 & 1 & 1 & 16 \\
\hline GX 301-2 & 681 & 2 & 2 & 16,24 \\
\hline GX 304-1 & 272 & 1(flat) & 1 & 25 \\
\hline 2S 1417-624 & 17.6 & 2 & 2 & 16 \\
\hline 4U 1538-52 & 530 & 2 & 2 & 16,21 \\
\hline XTE J1543-568 & 27.1 & 2 & & 26 \\
\hline SWIFT J1626.6-5156 & 15.4 & 1 & & 27 \\
\hline IGR J16358-4726 & 228 & 1 & & 28 \\
\hline IGR J16393-4643 & 912 & 2 & & 29 \\
\hline OAO $1657-415$ & 37.7 & 1 & 1 & 16 \\
\hline 1RXS J170849.0-400910 ${ }^{d}$ & 11.0 & 1 & 1 & 10 \\
\hline GPS $1722-363$ & 414 & 1 & 1 & 30 \\
\hline AX J1749.1-2733 & 132 & 2 & & 31 \\
\hline GRO J1750-27 & 4.45 & 1 & 1 & 16,32 \\
\hline SAX J1802.7-2017 & 140 & 2 & & 33 \\
\hline SGR $1806-20^{d}$ & 7.47 & 2 & & 34 \\
\hline Sct X-1 & 111 & 2 & 2 & 35 \\
\hline GS $1843+00$ & 29.5 & 2 & 2 & 36 \\
\hline GS $1843-024$ & 94.3 & 1 & 1 & 37 \\
\hline IGR J18483-0311 & 21.1 & 2 & & 38 \\
\hline XTE J1855-026 & 361 & 1 & 1 & 39 \\
\hline XTE J1858+034 & 221 & 1 & 1 & 40 \\
\hline SGR $1900+14^{d}$ & 5.16 & 2 & & 34 \\
\hline 4U 1901+03 & 2.76 & 2 & & 41 \\
\hline XTE J1906+09 & 89 & 2 & 1 & 42 \\
\hline 4U 1907+09 & 440 & 2 & 2 & 43 \\
\hline 4U 1908+075 & 605 & 2 & & 44 \\
\hline XTE J1946+274 & 15.8 & 2 & 2 & 45 \\
\hline KS 1947+300 & 18.7 & 1 & 1 & 46,47 \\
\hline
\end{tabular}


Table 1. continued.

\begin{tabular}{|c|c|c|c|c|}
\hline Name & $P_{\text {spin }}{ }^{a}$ & Pulses $^{b}$ & $\mathrm{~B} 03^{c}$ & Ref. \\
\hline SW J2000.6+3210 & 1056 & 1 & & 48 \\
\hline EXO 2030+375 & 42 & 2 & 2 & 16,49 \\
\hline GRO J2058+42 & 198 & 1 & 1 & 16 \\
\hline SAX J2103.5+4545 & 359 & 1 & 1 & 50 \\
\hline Cep X-4 & 66.2 & 2 & 2 & 51 \\
\hline $1 \mathrm{E} 2259+586^{d}$ & 6.98 & 2 & 2 & 52 \\
\hline
\end{tabular}

Notes. ${ }^{(a)}$ Pulsar spin period (s). ${ }^{(b)}$ Number of pulses in the profile. ${ }^{(c)}$ Classification by B03. ${ }^{(d)}$ Magnetars.

References. (1) Lamb et al. (2002); (2) Finger et al. (2001); (3) Laycock et al. (2002); (4) Corbet et al. (2001); (5) Santangelo et al. (1998); (6) Levine et al. (1993); (7) Macomb et al. (1999); (8) Hall et al. (2000); (9) Santangelo et al. (1999); (10) Kuiper et al. (2006); (11) Mereghetti et al. (2000); (12) Tsygankov et al. (2006); (13) Robba \& Warwick (1989); (14) Burderi et al. (1998); (15) Woo et al. (1996); (16) Bildsten et al. (1997); (17) McBride et al. (2006); (18) Corbet \& Peele (1997); (19) Reig \& Roche (1999b); (20) Aoki et al. (1992); (21) Mihara (1995); (22) Coe et al. (1994); (23) Burderi et al. (2000); (24) Koh et al. (1997); (25) McClintock et al. (1977); (26) in't Zand et al. (2001a); (27) Reig et al. (2008); (28) Lutovinov et al. (2005a); (29) Bodaghee et al. (2006); (30) Tawara et al. (1989); (31) Karasev et al. (2008); (32) Scott et al. (1997); (33) Augello et al. (2003); (34) Göğüş et al. (2002); (35) Koyama et al. (1991b); (36) Koyama et al. (1990); (37) Finger et al. (1999); (38) Sguera et al. (2007); (39) Corbet et al. (1999); (40) Paul \& Rao (1998); (41) Galloway et al. (2005); (42) Wilson et al. (2002); (43) in 't Zand et al. (1998); (44) Levine et al. (2004); (45) Wilson et al. (2003); (46) Tsygankov \& Lutovinov (2005); (47) Chakrabarty et al. (1995); (48) Morris et al. (2009); (49) Stollberg et al. (1999); (50) Hulleman et al. (1998); (51) Koyama et al. (1991a); (52) Iwasawa et al. (1992).

\section{Modeling light curves of X-ray pulsars}

\subsection{Model setup}

In order to obtain some constraints on the (distribution of) NS parameters such as compactness, magnetic field inclination, and the emissivity pattern from the statistical data (such as the fraction of the double-peaked profiles), we need to make a set of simplifying assumptions regarding the NS and the emission. In most of the following discussion, we assume that the NSs have a dipole magnetic field and two antipodal, point-like radiating hotspots at the magnetic poles at the NS surface. We assume also that all NS have the same compactness and that the emission from pulsars is described by the same pencil-beam pattern. As we will see below, the assumption of the same emission pattern for all pulsars will not have much effect on the results and therefore could be relaxed. Thus the pulsars differ from each other by the observer inclination, magnetic field inclination, and possibly by the emission pattern. We now discuss our assumptions one by one.

1. Compactness. The gravitational light-bending effect depends only on the compactness, i.e. mass-to-radius ratio $M / R$, which we assume to be the same for all NS. This is reasonable, because the observed distribution of NS masses in radio pulsars is very narrow (Thorsett \& Chakrabarty 1999; Haensel et al. 2007) and the accretion in high-mass systems could not provide a significant mass increase during the life time of the system. Below we will also show that the statistics of pulse profiles depends very little on the compactness for realistic emission patterns.
Table 2. Light curve classification of $64 \mathrm{X}$-ray pulsars observed at energies below $10 \mathrm{keV}$.

\begin{tabular}{|c|c|c|c|c|}
\hline Name & $P_{\text {spin }^{a}}{ }^{a}$ & Pulses $^{b}$ & $\mathrm{~B} 03^{c}$ & Ref. \\
\hline XMMU J004723.7-731226 & 263 & 1 & & 1 \\
\hline AX J0049-729 & 74.7 & 1 & 1 & 2 \\
\hline AX J0049-732 & 9.1 & 1 & 1 & 3 \\
\hline AX J0049.5-7323 & 756 & 1 & & 4 \\
\hline 2E $0050.1-7247$ & 8.9 & 1 & 1 & 5 \\
\hline RX J0051.3-7216 & 91 & 2 & & 6 \\
\hline AX J0051-733 & 323 & 1 & 1 & 7 \\
\hline AX J0051.6-7311 & 172 & 1 & & 8 \\
\hline SMC X-3 & 7.8 & 1 & & 9 \\
\hline XTE J0052-725 & 82.5 & 1 & & 9 \\
\hline XTE J0052-723 & 4.78 & 2 & & 10 \\
\hline CXOU J005323.8-722715 & 138 & 1 & & 9 \\
\hline RX J0053.8-7226 & 46.6 & 1 & & 11 \\
\hline XTE J0054-720 & 168 & 1 & & 12 \\
\hline CXOU J005455.6-724510 & 500 & 1 & & 9,13 \\
\hline RX J0054.9-7226 & 59 & 1 & & 14 \\
\hline XMMU J005517.9-723853 & 702 & 1 & & 13 \\
\hline CXOU J005527.9-721058 & 34.1 & 2 & & 9 \\
\hline XMMU J005605.2-722200 & 140 & 2 & & 14 \\
\hline AX J0057.4-7325 & 101 & 1 & & 15 \\
\hline CXOU J005736.2-721934 & 563 & 1 & & 9,18 \\
\hline RX J0057.8-7207 & 152 & 1 & & 14 \\
\hline AX J0058-720 & 281 & 2 & 2 & 14,16 \\
\hline 1XMMU J005921.0-722317 & 202 & 1 & & 1 \\
\hline RX J0059.2-7138 & 2.76 & 1 & 1 & 17 \\
\hline CXOU J010043.1-721134 ${ }^{d}$ & 8.02 & 2 & & 1 \\
\hline CXOU J010102.7-720658 & 304 & 1 & & 18 \\
\hline RX J0101.3-7211 & 452 & 1 & & 14 \\
\hline XTE J0103-728 & 6.85 & 2 & & 19 \\
\hline SAX J0103.2-7209 & 345 & 1 & 1 & 20 \\
\hline RX J0103.6-7201 & 1323 & 2 & & 21 \\
\hline J0105-721 & 3.34 & 1 & & 5 \\
\hline XTE J0111.2-7317 & 30.9 & 2 & 1 & 22 \\
\hline RX J0440.9+4431 & 202 & 1 & 1 & 23 \\
\hline RX J0502.9-6626 & 4.06 & 2 & 1 & 24 \\
\hline RX J0529.8-6556 & 69 & 1 & 1 & 25 \\
\hline XMMU J053011.2-655122 & 272 & 2 & & 26 \\
\hline EXO 053109-6609.2 & 13.7 & 2 & & 26 \\
\hline 1A $0538-66$ & 0.069 & 2 & 2 & 27 \\
\hline 1SAX J0544.1-710 & 96 & 2 & 1 & 28 \\
\hline SAX J0635.2+0533 & 0.034 & 1 & 1 & 29 \\
\hline RX J0648.1-4419 & 13.2 & 1 & 1 & 30 \\
\hline RX J0720.4-3125 & 8.39 & 1 & 1 & 31 \\
\hline RX J1037.5-5647 & 860 & 2 & 1 & 23 \\
\hline 1E $1048.1-5937^{d}$ & 6.44 & 1 & 1 & 32 \\
\hline IGR J11215-5952 & 187 & 2 & & 33 \\
\hline IGR J11435-6109 & 162 & 2 & & 34 \\
\hline 2RXP J130159.6-635806 & 710 & 1 & & 35 \\
\hline 1SAX J1324.4-6200 & 171 & 1 & 1 & 36 \\
\hline 1SAX J1452.8-5949 & 437 & 1 & 1 & 37 \\
\hline 2S $1553-54(2)$ & 9.3 & 2 & 2 & 38 \\
\hline IGR J16320-4751 & 1300 & 2 & & 39 \\
\hline IGR J16465-4507 & 228 & 1 & & 40 \\
\hline CXOU J164710.2-455216 ${ }^{d}$ & 10.6 & 2 & & 41 \\
\hline
\end{tabular}


Table 2. continued.

\begin{tabular}{lcccc}
\hline \hline Name & $P_{\text {spin }^{a}}{ }^{a}$ & Pulses $^{b}$ & B03 $^{c}$ & Ref. \\
\hline AX J170006-4157 & 715 & 1 & 1 & 42 \\
AX J1740.1-2847 & 730 & 2 & & 43 \\
AX J1749.2-2725 & 220 & 1 & 1 & 44 \\
XTE J1810-197 & 5.54 & 1 & & 45 \\
AX 1820.5-1434 & 152 & 2 & & 46 \\
XTE J1829-098 $^{2}$ & 7.8 & 1 & & 47 \\
AX J1841.0-0536 & 4.74 & 1 (flat) & & 48 \\
1E 1841-045 $^{d}$ & 11.8 & 1 & & 49 \\
AX J1845.0-0300 & 7.0 & 1 & 1 & 50 \\
SAX J2239.3+6116 & 1247 & 1 & & 51
\end{tabular}

Notes. ${ }^{(a)}$ Pulsar spin period (s). ${ }^{(b)}$ Number of pulses in the profile. ${ }^{(c)}$ Classification by B03. ${ }^{(d)}$ Magnetars.

References. (1) Majid et al. (2004); (2) Yokogawa et al. (1999); (3) Ueno et al. (2000); (4) Yokogawa et al. (2000b); (5) Israel et al. (1997a); (6) Yokogawa et al. (2000a); (7) Imanishi et al. (1999); (8) Yokogawa et al. (2000d); (9) Edge et al. (2004); (10) Laycock et al. (2003); (11) Laycock et al. (2005); (12) Yokogawa et al. (2001); (13) Haberl et al. (2004a); (14) Sasaki et al. (2003); (15) Yokogawa et al. (2000e); (16) Tsujimoto et al. (1999); (17) Kohno et al. (2000); (18) Macomb et al. (2003); (19) Haberl \& Pietsch (2008); (20) Israel et al. (2000); (21) Haberl \& Pietsch (2005); (22) Yokogawa et al. (2000c); (23) Reig \& Roche (1999a); (24) Schmidtke et al. (1995); (25) Haberl et al. (1997); (26) Haberl et al. (2003); (27) Skinner et al. (1982); (28) Cusumano et al. (1998); (29) Cusumano et al. (2000); (30) Israel et al. (1997b); (31) Haberl et al. (2004b); (32) Seward et al. (1986); (33) Sidoli et al. (2007); (34) in't Zand \& Heise (2004); (35) Chernyakova et al. (2005); (36) Angelini et al. (1998); (37) Oosterbroek et al. (1999); (38) Kelley et al. (1983); (39) Lutovinov et al. (2005b); (40) Lutovinov et al. (2005a); (41) Israel et al. (2007); (42) Torii et al. (1999); (43) Sakano et al. (2000); (44) Torii et al. (1998a); (45) Halpern \& Gotthelf (2005); (46) Kinugasa et al. (1998); (47) Halpern \& Gotthelf (2007); (48) Bamba et al. (2001); (49) Morii et al. (2003); (50) Torii et al. (1998b); (51) in't Zand et al. (2001b).

2. Point-like emission regions. The lower limit on the spot size in accreting X-ray pulsars can be obtained by assuming that the accreting matter is bound by the magnetic field lines intersecting the Alfvén radius (Wang \& Welter 1981). For a pulsar with mass-accretion rate $\dot{M}=10^{-9} M_{\odot} /$ year, magnetic field strength of $B=10^{12} \mathrm{G}$, and typical NS mass $M=1.4 M_{\odot}$ and radius $R=10 \mathrm{~km}$, the typical size of the hotspots is about 4 degrees. A study of the interchange instability and diffusion of plasma through the magnetic field gives a higher estimate of about 20 degrees (Arons \& Lea 1980). Thus in any case, the radiating spot size is much smaller than the stellar radius and the pulse profiles will not be dramatically affected because of the strong gravitational bending. Increasing the spot size increases the probability to see the secondary spot, but unless the emission pattern is fan-like, the number of pulses will not be affected.

For magnetars, the area of the thermally emitting region is only a few $\mathrm{km}^{2}$ (Mereghetti et al. 2002). Although the nature of the non-thermal persistent emission of anomalous X-ray pulsars and soft-gamma ray repeaters above $10 \mathrm{keV}$ (Kuiper et al. 2004; Molkov et al. 2005; Mereghetti et al. 2005; Kuiper et al. 2006) is not known, it also can be produced in very localized regions close to magnetic poles
(Thompson \& Beloborodov 2005; Beloborodov \& Thompson 2007). Thus the assumption of the spot-like regions seems reasonable and the corrections arising from a finite spot size are negligible.

3. Emission patterns. The exact geometry and the structure of the emission region in accreting X-ray pulsars is modeldependent and varies from plane-parallel slabs (see e.g. Nagel 1981a,b; Kirk et al. 1986; Meszaros \& Nagel 1985a,b) to columns/mounds (see e.g. Basko \& Sunyaev 1976; Burnard et al. 1991; Kraus 2001; Kraus et al. 2003; Leahy 2003; Becker \& Wolff 2005, and references therein). At high enough energies, where the pulse profiles are rather simple (see e.g. Nagase 1989; Bildsten et al. 1997), the effects of the cyclotron and photoelectric absorption are minimized. We parameterize the (possibly complicated) emission pattern with simple mathematical functions. For example, we take the pencil-like emission pattern with the surface flux given by $F \propto \cos ^{n} \alpha$, where $\alpha$ is the inclination of the spot normal to the light of sight. Detailed modeling of the pulse profiles of seven pulsars by Leahy \& Li (1995) showed that such a pattern with $n=2-4$ gives a good description of the data. These patterns also describe well theoretical dependences expected from a magnetized slab (Meszaros \& Nagel 1985 b) as was shown by Leahy (1990). The case $n=1$ corresponds to the blackbody-like emission. This emission pattern is probably not physical, but it is a useful starting point for discussing the pulsar classification scheme (Beloborodov 2002; Poutanen \& Beloborodov 2006). We also consider an alternative beaming pattern $F \propto \cos \alpha(1+h \cos \alpha)$, where $h>-1$ is a parameter. The case with $h<0$ would correspond to the scattering in an optically thin electron atmosphere associated with the accretion shock or heated NS surface layer (see e.g. Viironen \& Poutanen 2004), while $h>0$ resembles pencil-beam and is more appropriate for optically thick emission.

Although the physics of the persistent emission from magnetars is very different (Thompson \& Beloborodov 2005; Beloborodov \& Thompson 2007), the simplicity of their pulse profiles and their broad peaks argues in favor of broad emission beams, which can be represented by the assumed patterns.

We first assume that all pulsars can be described by the same emission pattern and then discuss the consequences of relaxing this assumption. We will show below that the statistics of pulse profiles depends very little on the actual emission pattern.

4. Antipodality. We assume that the hotspots are antipodal, even though many pulsars show asymmetric profiles. The detailed modeling of the pulse profiles (Leahy \& Li 1995; Kraus et al. 1996) shows, however, that the displacement of the spots relative to the antipodal position vary from a few to about 10 degrees. This displacement, while causing the profile asymmetry, does not change the number of pulses, which is important for our analysis.

5. Emission from the NS surface. Pulsars in Be-transient systems show time evolution in their pulse profiles during the outbursts related to the changing mass-accretion rate. This results either from variations in the emission pattern and/or changes in the accretion shock height. According to the recent cyclotron line measurements, the radiative region in accretion column does not extend higher than about $7 \%$ of the neutron star radius (Tsygankov et al. 2006). In magnetars the persistent emission most probably also originates from the stellar surface (Thompson \& Beloborodov 2005; 
M. Annala and J. Poutanen: Light curves of X-ray pulsars

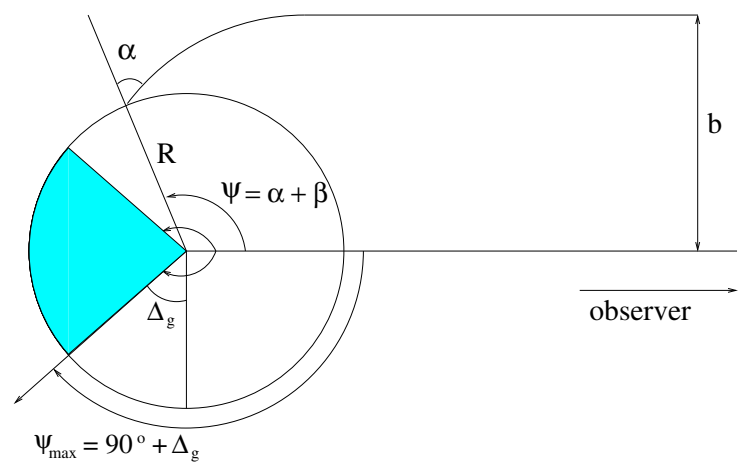

Fig. 1. Light emitted from a neutron star at an angle $\alpha$ to the normal is observed at impact parameter $b$, with the direction making angle $\psi$ to the spot position vector. The picture is in the plane of photon trajectory.

Beloborodov \& Thompson 2007). Therefore a simple model assuming the radiation is produced in the vicinity of the neutron star surface is justified.

6. Slow rotation. In principle, the stellar rotation could affect the profiles because of the effects of relativistic aberration and time delays (Poutanen \& Gierliński 2003; Poutanen \& Beloborodov 2006), but typical pulsars in Be-transient systems and high-mass X-ray binaries as well as magnetars rotate too slowly for these effects to be important.

7. Accretion disk. The accretion disk in strong magnetic field pulsars is normally disrupted at a distance that is large compared to the NS radius, and therefore it does not affect the visibility of the radiative spots on the neutron star surface or the pulse profile. These effects become important in NS in low-mass X-ray binaries such as accreting millisecond X-ray pulsars (see Poutanen 2008; Ibragimov \& Poutanen 2009), but we do not include these objects in our study.

\subsection{Pulsar classes and observed fluxes}

Let $\theta$ be the angle between rotational and magnetic axes and $i$ the inclination of the rotational axis to the line-of-sight. Then the unit vector in the observer's direction is $\boldsymbol{n}=(\sin i, 0, \cos i)$ and the unit vector in the direction of the primary spot from the NS center is $r=(\sin \theta \cos \varphi, \sin \theta \sin \varphi, \cos \theta)$ with $\varphi$ being the pulsar phase. As pulsar rotates the position of the spots relative to the observer changes. For the primary spot (closest to the observer)

$\cos \psi=\boldsymbol{n} \cdot \boldsymbol{r}=\cos \theta \cos i+\sin \theta \sin i \cos \varphi$.

At $\varphi=0$ the primary spot is closest to the observer and $\psi=$ $\psi_{\min }=i-\theta$, while at $\varphi=\pi$ the spot is farthest away and $\psi=$ $\psi_{\max }=i+\theta$.

Because of gravitational light bending, photons emitted at an angle $\alpha$ relative to the local radial direction reach the observer at angle $\psi=\alpha+\beta$ (see Fig. 1). The deflection angle $\beta$ reaches the maximum $\Delta_{\mathrm{g}}$ when photons are emitted at grazing angles $\alpha=\pi / 2$. This defines the visible part of the star:

$\psi<\psi_{\max }=\frac{\pi}{2}+\Delta_{\mathrm{g}}$,

where $\Delta_{\mathrm{g}}$ is a function of stellar compactness. For a typical neutron star mass of $1.4 M_{\odot}$ and radii between 10 and $14 \mathrm{~km}$, the maximum bending angle is between $45^{\circ}$ and $25^{\circ}$.

The relation between $\alpha$ and $\psi$ for slowly rotating stars is given by an elliptical integral (Pechenick et al. 1983). For stars with radii $R$ larger than about two Schwarzschild radii

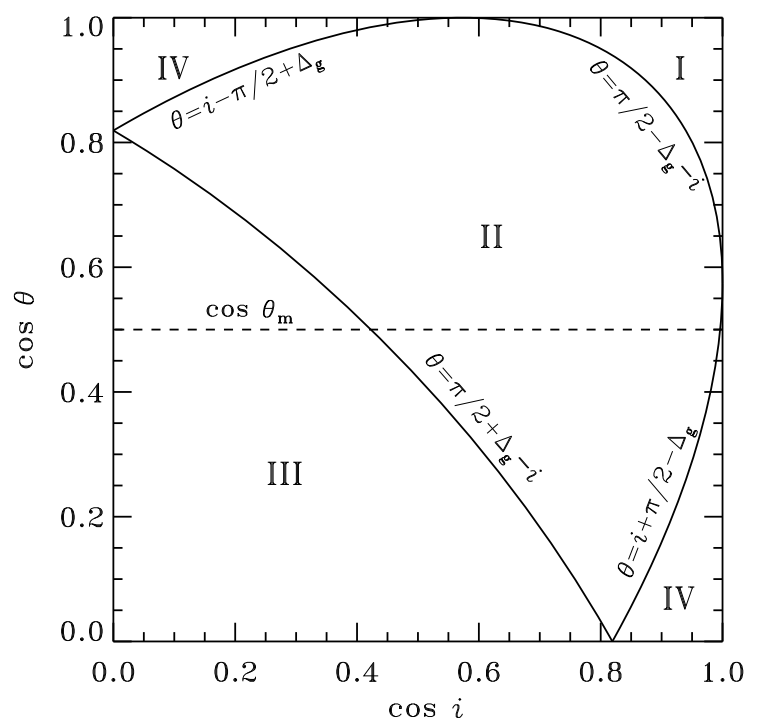

Fig. 2. Beloborodov's classes of pulsars on the plane $(\cos i, \cos \theta)$. The visibility classes I-IV are shown for a moderate light bending $\Delta_{\mathrm{g}}=30^{\circ}$. If the magnetic inclination is constrained by $\theta<\theta_{\mathrm{m}}$, the area below the dashed line $\cos \theta=\cos \theta_{\mathrm{m}}$ is forbidden.

$r_{\mathrm{s}}=2 G M / c^{2}$, a very accurate linear relation between the respective cosines can be used instead (Beloborodov 2002; Poutanen \& Beloborodov 2006):

$1-\cos \alpha \approx(1-u)(1-\cos \psi)$,

where $u=r_{\mathrm{s}} / R$. In this approximation, the visibility condition (4) gets a simple form

$\cos \psi>\cos \psi_{\max }=-\frac{u}{1-u}$,

where now

$\sin \Delta_{\mathrm{g}}=\frac{u}{1-u} \equiv \kappa$.

For the assumed dipole magnetic field with two antipodal spots, a light curve can belong to any of the four visibility classes introduced by Beloborodov (2002), according to the values of $i, \theta$, and $u$. For a Class I pulsar, the secondary pole is invisible and the primary pole is always visible. Class II pulsars have their primary pole always visible, but the secondary appears and disappears during the rotation period. In Class III pulsars both poles appear and disappear during the rotational period. Class IV pulsars have both their poles visible at all times. See Fig. 2 for an example of visibility classes for a moderate light bending.

For random inclination $i$ and magnetic inclination $\theta$, the probability densities $\mathrm{d} P / \mathrm{d} \cos i$ and $\mathrm{d} P / \mathrm{d} \cos \theta$ are constants. Therefore, the area covered by a certain Class on the $(\cos i, \cos \theta)$ plane directly gives the probability of a random pulsar belonging to that class. Depending on the stellar compactness, the area covered by each Class changes, and for example at high $u$, bending is strong and the area occupied by the Class IV pulsars grows.

For the blackbody emitting spot, the visibility Class directly defines the shape of the light curve (Beloborodov 2002), while for an arbitrary emissivity pattern the number of light curve classes can differ from the number of visibility classes.

The observed flux from a small homogeneous spot can be expressed as

$F=I \Omega$, 
where $I$ is the observed intensity and $\Omega$ is the solid angle covered by the spot on the observer's sky. The spot of area $S=$ $R^{2} \mathrm{~d} \cos \psi \mathrm{d} \phi$ seen at impact parameter $(b, b+\mathrm{d} b)$ occupies a solid angle $\Omega=b \mathrm{~d} b \mathrm{~d} \phi / D^{2}$, where $D$ is the distance to the observer. Owing to the gravitational redshift, the bolometric intensity is reduced from the emitted value $I_{0}$ to

$I=(1-u)^{2} I_{0}(\alpha)$,

where the emitted intensity can be a function of the emission angle $\alpha$. The impact parameter $b$ and the emission angle $\alpha$ are related by

$\sin \alpha=\frac{b}{R} \sqrt{1-u}$.

Combining the expressions above we get (Beloborodov 2002; Poutanen \& Beloborodov 2006):

$F=(1-u)^{2} I_{0}(\alpha) \frac{1}{1-u} \frac{\mathrm{d} \cos \alpha}{\mathrm{d} \cos \psi} \frac{S \cos \alpha}{D^{2}}$.

Using approximation (5), the flux takes the form

$F=(1-u)^{2} I_{0}(\alpha) \frac{S \cos \alpha}{D^{2}}$.

\subsection{Light curves and probabilities for blackbody spots}

Let us first assume that the hotspots emit blackbody radiation, i.e. $I_{0}(\alpha)=I_{0}=$ const. The flux from a spot is then simply

$F=(1-u)^{2} I_{0} \frac{S}{D^{2}}[u+(1-u) \cos \psi]$

Below we will use the flux normalized to $F_{0} \equiv(1-u)^{2} I_{0} S / D^{2}$, i.e. the flux from the primary spot is

$F_{\mathrm{p}}=u+(1-u) \cos \psi=Q+U \cos \varphi$,

where

$Q=u+(1-u) \cos \theta \cos i, \quad U=(1-u) \sin \theta \sin i$,

and for the secondary spot the flux is

$F_{\mathrm{s}}=\cos \alpha_{\mathrm{s}}=u-(1-u) \cos \psi=2 u-Q-U \cos \varphi$.

Obviously the flux and the variability amplitude depend on the stellar compactness and the position of the spot on the NS surface. As discussed by Beloborodov (2002), the light curves from Class I pulsars are single-peaked. In Class II the light curves are also single-peaked with a plateau between the pulses. Class III is the only one contributing the double-peaked light curves. Class IV pulsars produce flat light curves, because

$F_{\mathrm{p}}+F_{\mathrm{s}}=2 u=$ const.

In the blackbody case, the probability to observe double-peaked light curves depends only on the maximal gravitational lightbending angle $\Delta_{\mathrm{g}}$. Because only Class III pulsars produce double-peaked profiles, the probability to observe it is given by the area $\Sigma_{\text {III }}$ occupied by Class III on $\cos i-\cos \theta$ plane (see Fig. 2):

$P_{\mathrm{d}}\left(\Delta_{\mathrm{g}}\right)=\Sigma_{\mathrm{III}}=\int_{0}^{\cos \Delta_{\mathrm{g}}} \cos \left(\frac{\pi}{2}+\Delta_{\mathrm{g}}-i\right) \mathrm{d} \cos \mathrm{i}=\left(\frac{\pi}{4}-\frac{\Delta_{\mathrm{g}}}{2}\right) \cos \Delta_{\mathrm{g}}$,

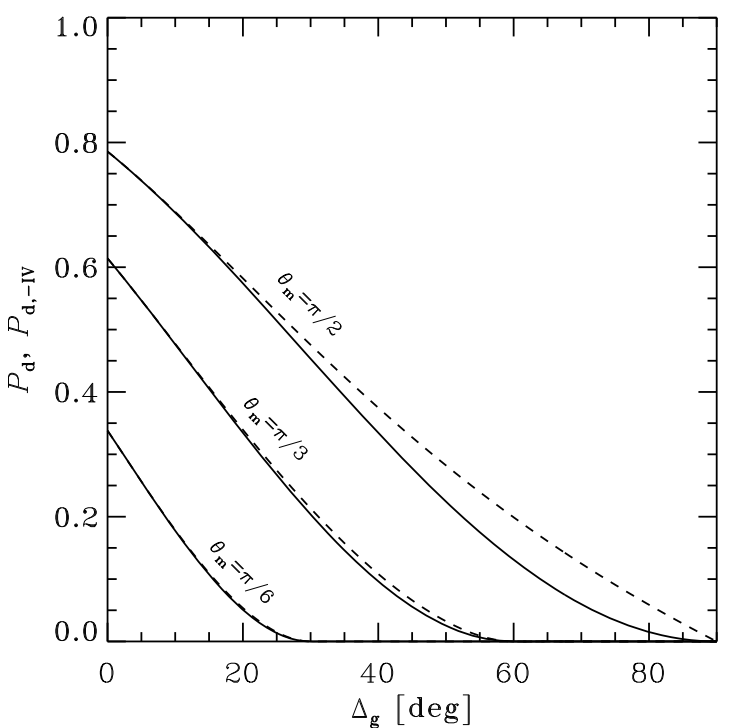

Fig. 3. Probability to obtain double-peaked light curves for blackbody spots as a function of the maximal gravitational light deflection angle $\Delta_{\mathrm{g}}$. Solid curves represent the probability given by Eq. (23) when class IV pulsars are included. The dashed curves represent the case where Class IV pulsars are excluded, given by Eq. (24). The curves correspond to three different cases of the maximum magnetic inclination $\theta_{\mathrm{m}}=\pi / 2$ (i.e. unconstrained), $\pi / 3$ and $\pi / 6$. The upper curves are given by analytical relations (20) and (18).

which is plotted as a solid curve in Fig. 3 (the case $\theta_{\mathrm{m}}=\pi / 2$ ). If bending is weak, $\Delta_{\mathrm{g}}=0$, the probability is $\pi / 4$, i.e. $79 \%$, and steadily decreases with increasing $\Delta_{\mathrm{g}}$. This result differs dramatically from that obtained by B03, who assumed that class II, III, and IV are all producing double-peaked profiles.

Another way to interpret the model predictions is to exclude the Class IV light curves from the light curve analysis since they are flat, i.e. these are not pulsars. It would mean that the total parameter space for the pulsars would be reduced and the fraction of single- and double-peaked light curves is changed (see Fig. 2). The area corresponding to the classes I, II, and III is

$$
\begin{aligned}
\Sigma_{\mathrm{I}+\mathrm{II}+\mathrm{III}}= & \int_{0}^{\sin \Delta_{\mathrm{g}}} \cos \left(i-\frac{\pi}{2}+\Delta_{\mathrm{g}}\right) \mathrm{d} \cos i+1-\sin \Delta_{\mathrm{g}} \\
& -\int_{\cos \Delta_{\mathrm{g}}}^{1} \cos \left(i+\frac{\pi}{2}-\Delta_{\mathrm{g}}\right) \mathrm{d} \cos i \\
= & 1-\sin \Delta_{\mathrm{g}}+\Delta_{\mathrm{g}} \cos \Delta_{\mathrm{g}},
\end{aligned}
$$

and the probability to observe a double-peaked light curve is thus

$P_{\mathrm{d},-\mathrm{IV}}\left(\Delta_{\mathrm{g}}\right)=\frac{\Sigma_{\mathrm{III}}}{\Sigma_{\mathrm{I}+\mathrm{II}+\mathrm{III}}}=\left(\frac{\pi}{4}-\frac{\Delta_{\mathrm{g}}}{2}\right)\left[\Delta_{\mathrm{g}}+\tan \left(\frac{\pi}{4}-\frac{\Delta_{\mathrm{g}}}{2}\right)\right]^{-1}$,

which is plotted as a function of the maximal gravitational light deflection angle in Fig. 3 (dashed curve, case $\theta_{\mathrm{m}}=\pi / 2$ ). This probability is only slightly higher than that given by Eq. (18) where Class IV is included (solid curve).

We need to notice here that Class IV pulsars produce flat light curves only if the emission is blackbody-like and if the radiating spots are exactly antipodal. Small deviations from the isotropy, e.g. if we use an Eddington approximation for the intensity, $I_{0}(\alpha)=I_{0}(1+h \cos \alpha)$ (where $h$ is a parameter), cause 

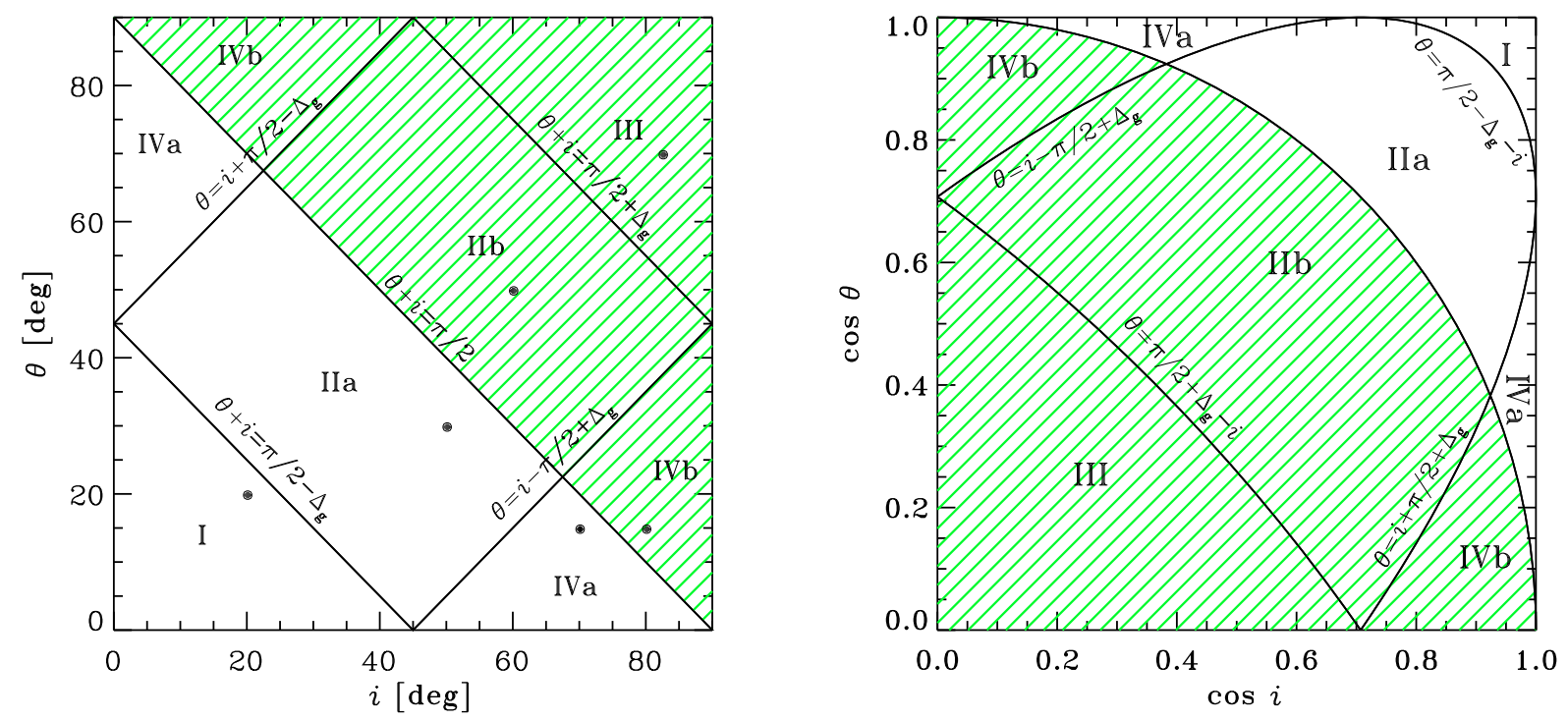

Fig. 4. Light curve classes for beam pattern $\cos ^{2} \alpha$ on the $i-\theta$ plane (left) and the $\cos i-\cos \theta$ plane (right). The shaded areas correspond to the double-peaked light curves. The number of light curve classes for this pattern differs from the blackbody case. The small filled circles at the left panel correspond to the parameter pairs $(i, \theta)$ of the light curves plotted in Fig. 5.

oscillations even in Class IV pulsars. For small $h$, the peak-topeak amplitude is (Poutanen \& Beloborodov 2006):

$A=\frac{F_{\max }-F_{\min }}{F_{\max }+F_{\min }}=\frac{1-u}{2 u}|h| \times \begin{cases}\sin 2 i \sin 2 \theta & \text { if } i+\theta<\pi / 2, \\ \cos ^{2}(i-\theta) & \text { if } i+\theta>\pi / 2 .\end{cases}$

In addition, even a slight displacement from the exactly antipodal positions leads to pulsation of the light curve. Let us assume the secondary spot is shifted by $\delta \theta$ and $\delta \varphi$ in latitude and azimuth: $\theta_{\mathrm{s}}=\pi-(\theta+\delta \theta)$ and $\varphi_{\mathrm{s}}=\varphi+\pi+\delta \varphi$. It is easy to show that for the blackbody emission

$A=\frac{1-u}{2 u} \sin i \sqrt{\cos ^{2} \theta(\delta \theta)^{2}+\sin ^{2} \theta(\delta \varphi)^{2}}$.

Thus we see that the Class IV pulsars are actually expected to pulsate. They predominantly have single-peaked profiles if the radiation emission pattern is close to black body (or more peaked, see Sects. 3.4.1 and 3.4.2), and spots are close to antipodal positions. Equation (18) should thus give a more realistic probability of observing double-peaked light curves.

\subsubsection{Limiting magnetic inclination}

In the previous discussion, we assumed that magnetic inclination can take any value $\theta \leq \pi / 2$. However, as proposed by Wang $\&$ Welter (1981) and B03, there can be an upper limit to that angle $\theta_{\mathrm{m}}$. Then the fraction of pulsars belonging to different visibility classes is changed (see Fig. 2). The constrained $\theta$ case alters the probabilities of observing the double-peaked profiles.

Let us consider a blackbody-like emission pattern and include all the pulsar classes I-IV. The probability to observe double-peaked light curves is proportional to the area of class III above the curve $\cos \theta=\cos \theta_{\mathrm{m}}$ (see Fig. 2) and is the function of the bending angle $\Delta_{\mathrm{g}}$ and $\theta_{\mathrm{m}}$ :

$$
\begin{aligned}
P_{\mathrm{d}}\left(\Delta_{\mathrm{g}}, \theta_{\mathrm{m}}\right) & =\frac{\int_{0}^{\cos i_{\min }} \cos \left(\frac{\pi}{2}+\Delta_{\mathrm{g}}-i\right) \mathrm{d} \cos i-\cos \theta_{\mathrm{m}} \cos i_{\min }}{1-\cos \theta_{\mathrm{m}}} \\
& =\frac{\left(\theta_{\mathrm{m}}-\Delta_{\mathrm{g}}\right) \cos \Delta_{\mathrm{g}}+\cos \theta_{\mathrm{m}} \sin \left(\Delta_{\mathrm{g}}-\theta_{\mathrm{m}}\right)}{2\left(1-\cos \theta_{\mathrm{m}}\right)},
\end{aligned}
$$

where $i_{\min }=\pi / 2+\Delta_{\mathrm{g}}-\theta_{\mathrm{m}}$ is the crossing point between curves $\theta=\theta_{\mathrm{m}}$ and $\theta=\pi / 2+\Delta_{\mathrm{g}}-i$. If $\theta_{\mathrm{m}}<\Delta_{\mathrm{g}}$, the probability is zero.

If we exclude Class IV from consideration, the corresponding probability becomes

$$
P_{\mathrm{d},-\mathrm{IV}}\left(\Delta_{\mathrm{g}}, \theta_{\mathrm{m}}\right)= \begin{cases}0 & \text { if } \theta_{\mathrm{m}}<\Delta_{\mathrm{g}} \\ P_{1} & \text { if } \Delta_{\mathrm{g}}<\theta_{\mathrm{m}}<\frac{\pi}{2}-\Delta_{\mathrm{g}} \text { and } \Delta_{\mathrm{g}}<\frac{\pi}{4} \\ P_{2} & \text { if } \frac{\pi}{2}-\Delta_{\mathrm{g}}<\theta_{\mathrm{m}}<\frac{\pi}{2} \text { and } \Delta_{\mathrm{g}}<\frac{\pi}{4} \\ P_{2} & \text { if } \Delta_{\mathrm{g}}<\theta_{\mathrm{m}}<\frac{\pi}{2} \text { and } \Delta_{\mathrm{g}}>\frac{\pi}{4},\end{cases}
$$

where

$P_{1}=\frac{\left(\theta_{\mathrm{m}}-\Delta_{\mathrm{g}}\right) \cos \Delta_{\mathrm{g}}+\cos \theta_{\mathrm{m}} \sin \left(\Delta_{\mathrm{g}}-\theta_{\mathrm{m}}\right)}{2\left(1-\cos \theta_{\mathrm{m}}\right)-\left(\sin \Delta_{\mathrm{g}}-\Delta_{\mathrm{g}} \cos \Delta_{\mathrm{g}}\right)}$

and

$$
P_{2}=\frac{\left(\theta_{\mathrm{m}}-\Delta_{\mathrm{g}}\right) \cos \Delta_{\mathrm{g}}+\cos \theta_{\mathrm{m}} \sin \left(\Delta_{\mathrm{g}}-\theta_{\mathrm{m}}\right)}{2\left(1-\sin \Delta_{\mathrm{g}}\right)+\left(2 \Delta_{\mathrm{g}}-\frac{\pi}{2}+\theta_{\mathrm{m}}\right) \cos \Delta_{\mathrm{g}}-\cos \theta_{\mathrm{m}} \sin \left(\Delta_{\mathrm{g}}+\theta_{\mathrm{m}}\right)} \cdot(2
$$

The probabilities to observe double-peaked profiles for two cases, including and excluding the Class IV pulsars, given by Eqs. (23) and (24), are shown in Fig. 3 by solid and dashed curves, respectively. The significant difference in these probabilities appears only for a large bending angle $\Delta_{\mathrm{g}}$.

\subsection{Modified pencil beam pattern}

\subsubsection{Beam pattern $\cos ^{2} \alpha$}

Let us now consider two point-like antipodal spots with the emitted intensity given by $I_{0}(\alpha)=I_{0} \cos \alpha$. The light curves produced by the two spots with this more radially concentrated beampattern $\cos ^{2} \alpha$ can be divided into six different classes based on the visibility of the spots and whether the light curves are singleor double-peaked (see Figs. 4 and 5). The normalized flux from the primary spot in Beloborodov's approximation (5) is

$F_{\mathrm{p}}=\cos ^{2} \alpha=(Q+U \cos \varphi)^{2}$.

The flux from the secondary pole is

$F_{\mathrm{s}}=\cos ^{2} \alpha_{\mathrm{s}}=(2 u-Q-U \cos \varphi)^{2}$. 


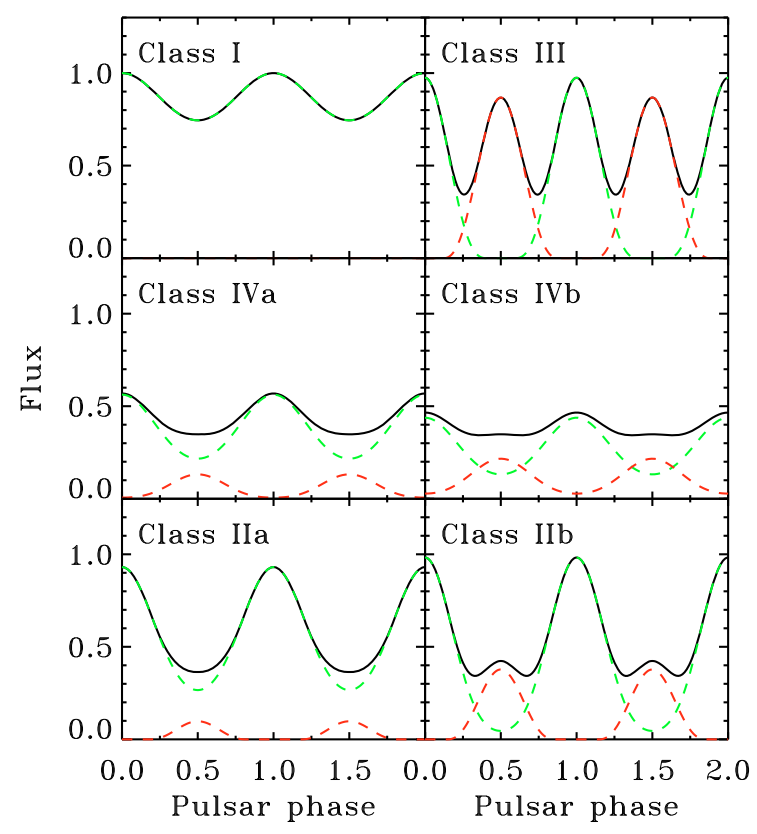

Fig. 5. Light curve classes for beam pattern $\cos ^{2} \alpha$ in the moderate case of light bending $\Delta_{\mathrm{g}}=45^{\circ}$. The light curves are plotted with following parameters: $\theta=20^{\circ}$ and $i=20^{\circ}$ (class I), $\theta=70^{\circ}$ and $i=82^{\circ}$ (class III), $\theta=15^{\circ}$ and $i=70^{\circ}$ (class IVa), $\theta=15^{\circ}$ and $i=80^{\circ}$ (Class IVb), $\theta=30^{\circ}$ and $i=50^{\circ}$ (class IIa), $\theta=50^{\circ}$ and $i=60^{\circ}$ (class IIb).

In Class I, only the primary spot is visible and the light curve is single-peaked with the maximum at pulsar phase $\varphi=0$ and the minimum at $\varphi=\pi$. In Class IV, both spots are visible all the time and the flux $F_{\mathrm{p}}+F_{\mathrm{s}}$ has local extrema when

$\frac{\mathrm{d}\left(F_{\mathrm{p}}+F_{\mathrm{s}}\right)}{\mathrm{d} \varphi}=4 U \sin \varphi(u-Q-U \cos \varphi)=0$,

i.e. at $\varphi=0, \pi$, or

$\cos \varphi=\cos \varphi_{\min } \equiv \frac{u-Q}{U}=\cot i \cot \theta$.

The latter extrema exist only when

$i+\theta>\pi / 2$

and then the light curve is double-peaked. Similarly for Class II pulsars, for which the primary spot is always visible and the secondary spot is seen at phases around $\varphi \sim \pi$, we get that the light curve is single-peaked when $i+\theta<\pi / 2$ and double-peaked otherwise. Condition (31) divides both classes II and IV into two subclasses according to the number of peaks: in IIa and IVa profiles are single-peaked, while in classes IIb and IVb, they are double-peaked. In Class III, both spots appear and disappear from the view at some pulsar phases, and it is easy to show that both $\varphi=0$ and $\varphi=\pi$ are maxima, and the light curve is always double-peaked.

Thus the light curve in classes I, IIa, and IVa is single-peaked and the profile in III, IIb, and IVb is double-peaked. The probability to observe double-peaked light curves is now independent of the neutron star compactness (see the hatched area in Fig. 4):

$P_{\mathrm{d}}=\int_{0}^{1} \cos \left(\frac{\pi}{2}-i\right) \mathrm{d} \cos i=\frac{\pi}{4} \approx 0.79$.

This coincides with expression (18) for the Newtonian case, $\Delta_{\mathrm{g}}=0$, and the blackbody radiation pattern.

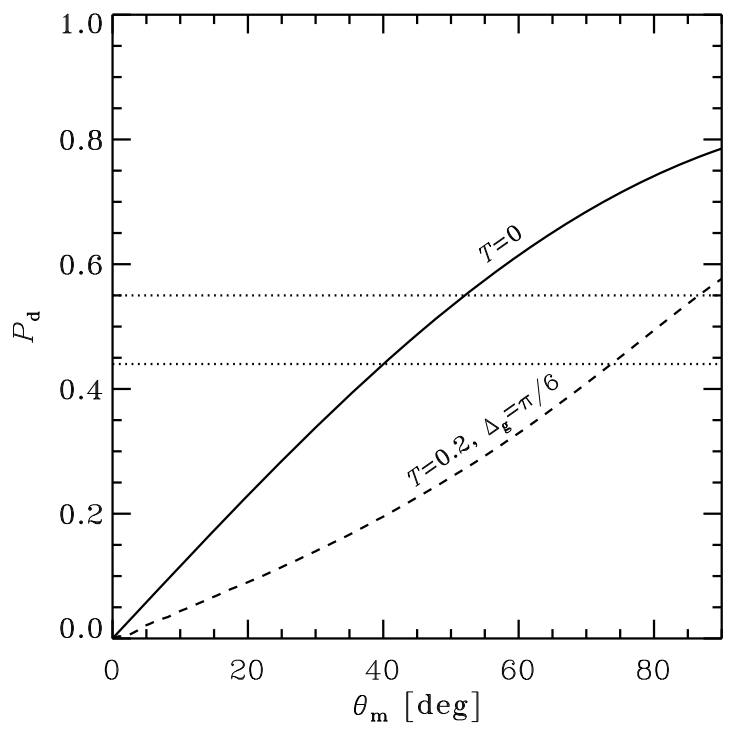

Fig. 6. Probability to observe double-peaked light curves as a function of the maximum magnetic inclination $\theta_{\mathrm{m}}$. The solid curve corresponds to any modified pencil beam patterns discussed in Sect. 3.4, $\cos ^{n} \alpha$ and $\cos \alpha(1+h \cos \alpha)$ (Eq. (33)) in the case of full detectability of the secondary pulse, $T=0$. The dashed curve shows an example of the beam pattern $\cos ^{2} \alpha$ with non-zero threshold $T=0.2$ and a moderate bending angle $\Delta_{\mathrm{g}}=30^{\circ}$. The dotted lines correspond to the observed probability of having double-peaked profiles as given by the classification of data $p_{0}=0.44$ and $p_{1}=0.55$ (see Sect. 2).

If the magnetic inclination $\theta$ is constrained to lie in the interval $\left(0, \theta_{\mathrm{m}}\right)$, the probability to observe a double-peaked light curve is (shown by the solid curve in Fig. 6)

$P_{\mathrm{d}}\left(\theta_{\mathrm{m}}\right)=\frac{1}{4} \frac{2 \theta_{\mathrm{m}}-\sin 2 \theta_{\mathrm{m}}}{1-\cos \theta_{\mathrm{m}}}$

Decreasing the value of $\theta_{\mathrm{m}}$ reduces the probability to obtain double-peaked light curves and for $\theta_{\mathrm{m}} \ll 1, P_{\mathrm{d}}\left(\theta_{\mathrm{m}}\right) \approx \frac{2}{3} \theta_{\mathrm{m}}$.

\subsubsection{Beam pattern $\cos ^{n} \alpha$}

Let us assume that the emission pattern can be described as $I_{0}(\alpha)=I_{0} \cos ^{n-1} \alpha$, where $n \geq 1$. The observed flux is then $F \propto I_{0}(\alpha) \cos \alpha=I_{0} \cos ^{n} \alpha$. The normalized flux from the primary pole is then

$F_{\mathrm{p}}=\cos ^{n} \alpha=(Q+U \cos \varphi)^{n}$

and the secondary pole has the flux

$F_{\mathrm{s}}=\cos ^{n} \alpha_{\mathrm{s}}=(2 u-Q-U \cos \varphi)^{n}$.

Once the power law index $n$ exceeds 1 , the light curves become similar to the case of $n=2$, i.e. the $\cos ^{2} \alpha$ beam pattern. Doublepeaked profiles are obtained in the region $i+\theta>\pi / 2$. Therefore the probability to observe double-peaked light curves in the case of $n>1$ and randomly distributed angles $i$ and $\theta$ is $P_{\mathrm{d}}=\pi / 4$. For the constrained magnetic dipole inclination, this probability is given by Eq. (33).

\subsubsection{Eddington approximation $\cos \alpha(1+\mathrm{h} \cos \alpha)$}

So far we have considered modified pencil beam patterns with varying power index $n$. Let us now assume the radiation intensity deviates from blackbody according to the Eddington 
approximation $I_{0}(\alpha)=I_{0}(1+h \cos \alpha)$ and the observed flux $F \propto I_{0}(\alpha) \cos \alpha=I_{0} \cos \alpha(1+h \cos \alpha)$. The normalized primary pole flux is

$F_{\mathrm{P}}=(Q+U \cos \varphi)[1+h(Q+U \cos \varphi)]$

and the secondary pole gives

$F_{\mathrm{S}}=(2 u-Q-U \cos \varphi)[1+h(2 u-Q-U \cos \varphi)]$.

The shape of the light curves is determined by the anisotropy parameter $h$. The observations show that $h$ should be positive rather than negative, because the negative values can produce double-, triple- and quadruple-peaked light curves, which are not consistent with the observations. Even if there are only single- and double-peaked light curves (for example when $h=-1$ ), these double-peaked light curves are double-horned with equal primary and secondary peak amplitudes. This is not what we generally observe.

Therefore, we consider positive values of $h$. The light curves then resemble the classes obtained for modified beam pattern $\cos ^{n} \alpha$. Double-peaked light curves are only obtained when $i+\theta>\pi / 2$ and single-peaked curves when $i+\theta<\pi / 2$. Again, this result does not depend on the compactness. Therefore, if angles $\theta$ and $i$ can vary randomly between zero and $\pi / 2$, we would observe about $79 \%$ double-peaked light curves as calculated earlier (see Eq. (32)). If the angle between the magnetic field and rotational axis is constrained, then the probability of having doublepeaked light curves is just a function of $\theta_{\mathrm{m}}$ as found earlier (see Eq. (33)). As can be seen, the probabilities to observe doublepeaked light curves do not depend on the constant $h$ when it is positive.

\subsection{Effect of the detection threshold}

Limited photon statistics and (red-noise) flux variability can affect the visual assignment of pulsars into single- and doublepeaked classes. If the secondary pulse is hardly visible above the constant emission level, the pulsar is likely to be classified as single-peaked. It is difficult to quantify these effects. Following B03, we can assume that the secondary pulse is visible if its strength above the minimum flux is at least fraction $T$ of the strength of the primary maximum. The probability to observe double-peaked profiles decreases with increasing detection threshold $T$.

\subsubsection{Detection threshold in the blackbody case}

In the blackbody case, for the zero threshold $T=0$ the region, where profiles are double-peaked, coincides with Class III. There the primary maximum is reached at phase $\varphi=0$, and its amplitude above the constant flux level is $F_{\mathrm{p}}(\varphi=0)-2 u$ (see Eq. (17)). For the secondary maximum to be detected, its amplitude, $F_{\mathrm{s}}(\varphi=\pi)-2 u$, should exceed the threshold $T \times\left[F_{\mathrm{p}}(\varphi=\right.$ $0)-2 u$ ]. Thus for the non-zero $T$, the region of double-peaked profiles is reduced as the following condition must be satisfied

$\frac{F_{\mathrm{s}}(\varphi=\pi)-2 u}{F_{\mathrm{p}}(\varphi=0)-2 u}=\frac{\kappa+\cos (i+\theta)}{\kappa-\cos (i-\theta)}>T$.

Generally this inequality must be solved numerically. The resulting constraints are shown in Fig. 7a.

In the Newtonian limit $\Delta_{\mathrm{g}}=0$ (i.e. $\kappa=0$ ), this inequality is reduced to

$\tan i \tan \theta>\frac{1+T}{1-T}$.
Then the probability to observe double-peaked light curves for randomly distributed $i$ and $\theta$ is given by the integral

$$
\begin{aligned}
P_{\mathrm{d}} & =\int_{0}^{1} \cos \theta \mathrm{d} \cos i=\int_{0}^{1} \frac{\mathrm{d} \cos i}{\sqrt{1+\left(\frac{1+T}{1-T}\right)^{2} \cot ^{2} i}} \\
& =\frac{1-T^{2}}{4 T}[F(k)-E(k)],
\end{aligned}
$$

where $k=\sqrt{4 T} /(1+T), F(k)$ and $E(k)$ are the complete elliptic integrals.

Figure 7a demonstrates how the parameter space is reduced where profiles are double-peaked when the detection threshold is increased from zero to $T=0.2$ in a Newtonian $\left(\Delta_{\mathrm{g}}=0\right)$ and in a moderately relativistic case, $\Delta_{\mathrm{g}}=30^{\circ}$. The solid curves correspond to the total detectability $T=0$ and the areas below them correspond to the probabilities to observe double-peaked light curves. The dashed curves correspond to the threshold $T=$ 0.2 .

\subsubsection{Detection threshold for $\cos ^{n} \alpha$ beam pattern}

Let us consider the beam pattern $\cos ^{n} \alpha$, where the index $n$ is greater than 1. We showed in Sect. 3.4.2 that the resulting profile becomes double-peaked when $i+\theta>\pi / 2$. The effect of the detection threshold on the visibility of the secondary pulse has to be considered in three different light curve classes IIb, III, and IVb (see Fig. 4).

For a blackbody pattern when both poles are visible the flux is constant and equals $2 u$. For $n>1$, the minimum flux of $2 u^{n}$ is reached at the phase $\varphi_{\min }$ given by Eq. (30) in all three considered classes (because the secondary pole is visible at this phase).

For Class IIb, the primary maximum is reached at $\varphi=0$, when only the primary pole is visible. On the other hand, both poles contribute to the flux at the secondary maximum $\varphi=\pi$. Thus the secondary pulse will be detected if

$$
\frac{F_{\mathrm{p}}(\varphi=\pi)+F_{\mathrm{s}}(\varphi=\pi)-2 u^{n}}{F_{\mathrm{p}}(\varphi=0)-2 u^{n}}=\frac{(Q-U)^{n}+(2 u-Q+U)^{n}-2 u^{n}}{(Q+U)^{n}-2 u^{n}}>T .
$$

For Class III, the primary maximum is produced by the primary pole and the secondary maximum by the secondary pole only, thus the condition for the secondary pulse to be seen is

$$
\frac{F_{\mathrm{S}}(\varphi=\pi)-2 u^{n}}{F_{\mathrm{p}}(\varphi=0)-2 u^{n}}=\frac{(2 u-Q+U)^{n}-2 u^{n}}{(Q+U)^{n}-2 u^{n}}>T .
$$

Class IV pulsars have both their primary and secondary poles contributing to the flux all the time, and therefore the threshold to the secondary pulse to be detected is

$$
\begin{aligned}
& \frac{F_{\mathrm{p}}(\varphi=\pi)+F_{\mathrm{s}}(\varphi=\pi)-2 u^{n}}{F_{\mathrm{p}}(\varphi=0)+F_{\mathrm{s}}(\varphi=0)-2 u^{n}}= \\
& \frac{(Q-U)^{n}+(2 u-Q+U)^{n}-2 u^{n}}{(Q+U)^{n}+(2 u-Q-U)^{n}-2 u^{n}}>T .
\end{aligned}
$$

For $n=2$ the boundary between single- and double-peaked profiles given by relations (41)-(43) are shown in Fig. $7 \mathrm{~b}$ at the $\cos i-\cos \theta$ plane. The areas below the corresponding curves give the probability $P_{\mathrm{d}}$ to observe double-peaked profiles. Figure $8 \mathrm{a}$ shows this probability as a function of compactness for various indices $n$ and two thresholds $T=0$ and 0.2. When $T=0$, i.e. 

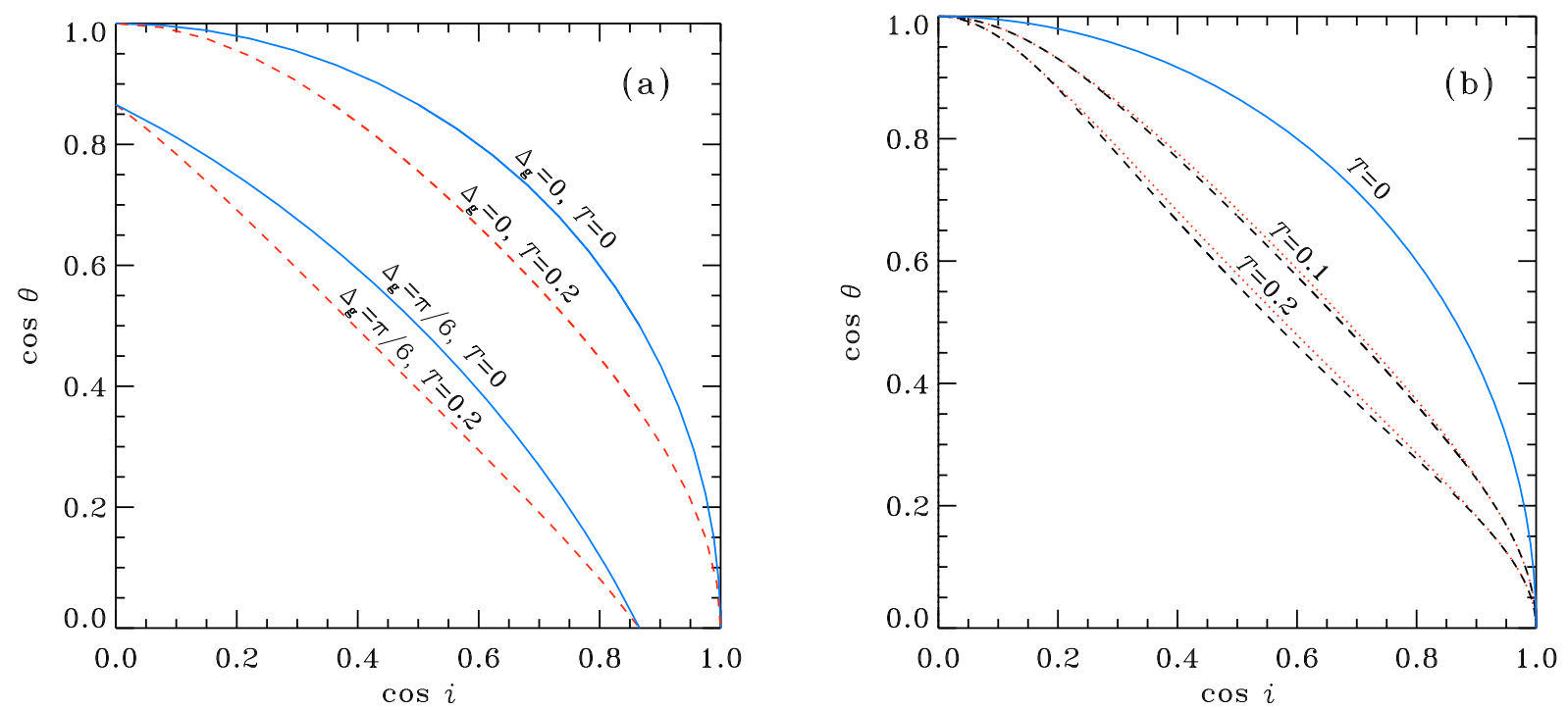

Fig. 7. Effect of the detection threshold on the fraction of the double-peaked profiles. a) The parameter space at the $\cos i-\cos \theta$ plane for the blackbody emission pattern. Upper curves are for the Newtonian case $\Delta_{\mathrm{g}}=0$ and the lower curves are for the moderate light bending $\Delta_{\mathrm{g}}=30^{\circ}$. The solid curves correspond to the zero threshold and the dashed curves are for $T=0.2$. The areas below respective curves correspond to the probability of observing double-peaked profiles. b) Same as a), but for the pencil-beam $\cos ^{2} \alpha$. For the zero threshold the curve is the same for any neutron star compactness. The curves for thresholds $T=0.1$ and 0.2 are shown by dotted and dashed curves, respectively. The dotted curves are for $\Delta_{\mathrm{g}}=30^{\circ}$, while the dashed ones are for $\Delta_{\mathrm{g}}=45^{\circ}$. Even then the dependence on compactness is very weak.
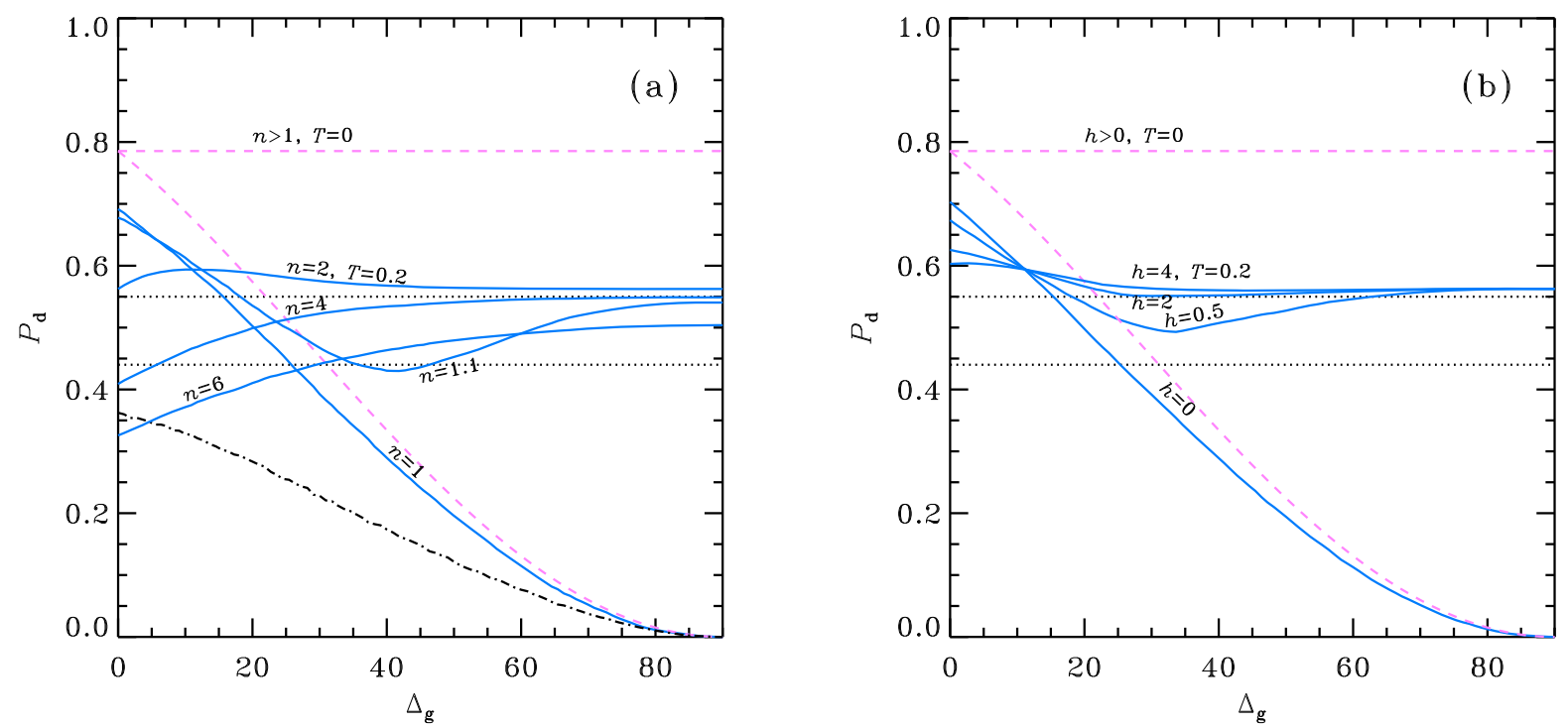

Fig. 8. a) Probability to observe double-peaked profiles for the blackbody emission pattern $(n=1)$ as well as for beam patterns with indices $n=1.1,2,4$ and 6 for two different threshold cases $T=0$ and 0.2. The dashed curves correspond to $T=0$, while the solid curves are for $T=0.2$. The dotted lines correspond to the observed probability of having double-peaked profiles as given by the classification of data $p_{0}=0.44$ and $p_{1}=0.55$ (see Sect. 2). The dash-dotted line correspond to the probability to have multiple-peaked light curves for two randomly positioned spots radiating as black bodies. b) Same as panel a), but for beam patterns in the Eddington approximation. Cases with anisotropy parameter $h=0,0.5$, 2 and 4 for two different thresholds $T=0$ and 0.2 are shown.

even the smallest bumps in the light curve can be detected, all beam patterns with $n>1$ predict $P_{\mathrm{d}}=79 \%$. For $T=0.2$, the probability to observe double-peaked profiles decreases. For typical $\Delta_{\mathrm{g}} \in\left[25^{\circ}, 45^{\circ}\right]$, a slight deviation from $n=1$ to $n=1.1$ immediately increases the value of $P_{\mathrm{d}}$ from $\sim 0.3$ to 0.45 . The maximum of $P_{\mathrm{d}} \sim 0.6$ is reached at $n \sim 2$, and at $n>2$ the probability starts decreasing again. This probability depends weakly on $\Delta_{\mathrm{g}}$ as well as on index $n$ (see Figs. $7 \mathrm{~b}$ and $8 \mathrm{a}$ ). If the magnetic inclination is constrained, the fraction of double-peaked profiles decreases as shown in Fig. 6 for the $n=2$ case.

\subsubsection{Detection threshold in the Eddington approximation case}

The emission pattern in the form given by the Eddington approximation produces light curve classes identical to the classes for the modified pencil beam $\cos ^{n} \alpha$. Therefore the consideration of the threshold is similar to the previous section. Double-peaked light curves are obtained only in classes IIb, III, and IVb, and increasing the threshold will increase the probability of observing single-peaked light curves in these classes. 
The local extrema are at the same pulsar phases as for the $\cos ^{2} \alpha$ beam pattern, i.e. at $\varphi=0, \pi$ and $\cos \varphi=(u-Q) / U$. At the minima the flux is

$F_{\mathrm{P}}\left(\cos \varphi=\frac{u-Q}{U}\right)+F_{\mathrm{S}}\left(\cos \varphi=\frac{u-Q}{U}\right)=2 u(1+h u)$.

In Class IVb, the secondary pulse is detected if the following condition holds:

$\frac{F_{\mathrm{p}}(\varphi=\pi)+F_{\mathrm{s}}(\varphi=\pi)-2 u(1+h u)}{F_{\mathrm{p}}(\varphi=0)+F_{\mathrm{s}}(\varphi=0)-2 u(1+h u)}=\frac{\cos ^{2}(i+\theta)}{\cos ^{2}(i-\theta)}>T$.

In Class IIb, the secondary pulse is detected if

$$
\begin{aligned}
& \frac{F_{\mathrm{p}}(\varphi=\pi)+F_{\mathrm{s}}(\varphi=\pi)-2 u(1+h u)}{F_{\mathrm{p}}(\varphi=0)-2 u(1+h u)}= \\
& \quad \frac{h\left[(Q-U)^{2}+(2 u-Q+U)^{2}-2 u^{2}\right]}{Q+U-2 u(1+h u)+h(Q+U)^{2}}>T .
\end{aligned}
$$

Finally in Class III, the secondary pulse is detected if

$$
\begin{aligned}
\frac{F_{\mathrm{s}}(\varphi=}{F_{\mathrm{p}}(\varphi)}= & 0)-2 u(1+h u) \\
& \frac{-Q+U+h(1+h u)}{Q+U+h(Q+U)^{2}-2 u(1+h u)}>T .
\end{aligned}
$$

The influence of the increasing threshold on the probability to observe double-peaked light curves for various anisotropy parameters $h$ is shown in Fig. 8b. The case $h=0$ corresponds to the blackbody emission pattern and therefore gives the same dependences as plotted in Fig. 8a (curves marked with $n=1$ ). With full detectability of the secondary pulse $T=0$ the probability to observe double-peaked profiles is constant $P_{\mathrm{d}} \approx 79 \%$ for any $h>0$. This is similar to the result obtained for the modified pencil beam pattern $\cos ^{n} \alpha$ with index $n>1$ discussed in Sect. 3.5.2.

When the threshold $T$ is increased, the probability to have double-peaked light curves falls and becomes almost independent of the bending angle $\Delta_{\mathrm{g}}$ and anisotropy parameter $h$.

\section{Results}

Based on our classification of pulsars into single- and doublepeaked in Sect. 2, we can now compare the corresponding fractions with the predictions of various radiation models.

\subsection{Blackbody emission pattern and comparison to B03}

Let us consider first the blackbody radiation pattern and assume that secondary pulses are fully detectable (i.e. $T=0$ ). If the magnetic inclination is unconstrained (i.e. $\theta_{\mathrm{m}}=\pi / 2$ ), taking $p=$ $p_{0}$ (Eq. (1)) we get the maximum deflection angle $\Delta_{\mathrm{g}}=31^{\circ} \pm 3^{\circ}$ ( $1 \sigma$ error), if we include Class IV pulsars into consideration, or $\Delta_{\mathrm{g}}=33^{\circ} \pm 4^{\circ}$ if we do not, see upper curves in Fig. 3. As the effect of excluding Class IV pulsars is small, we take below that Class into consideration. If we assume that there is an upper limit of magnetic inclination $\theta_{\mathrm{m}}$, we get that $\theta_{\mathrm{m}}>32^{\circ}$ (at $95 \%$ confidence) for any deflection angle, and for the Newtonian case (i.e. $\Delta_{\mathrm{g}}=0$ ) we have $\theta_{\mathrm{m}}=40^{\circ} \pm 4^{\circ}$. In general, the probability to observe a double-peaked profile was found to be given by Eq. (23), and the corresponding constraints on both $\theta_{\mathrm{m}}$ and $\Delta_{\mathrm{g}}$ are shown Fig. 9. For typical neutron stars with $\Delta_{\mathrm{g}} \in\left[25^{\circ}, 45^{\circ}\right]$, we have $\theta_{\mathrm{m}}>70^{\circ}$ (at $95 \%$ confidence). Thus our results favor not very compact neutron stars (with radius of above $12 \mathrm{~km}$ for $M=1.4 M_{\odot}$ ) and nearly random magnetic dipole inclinations.

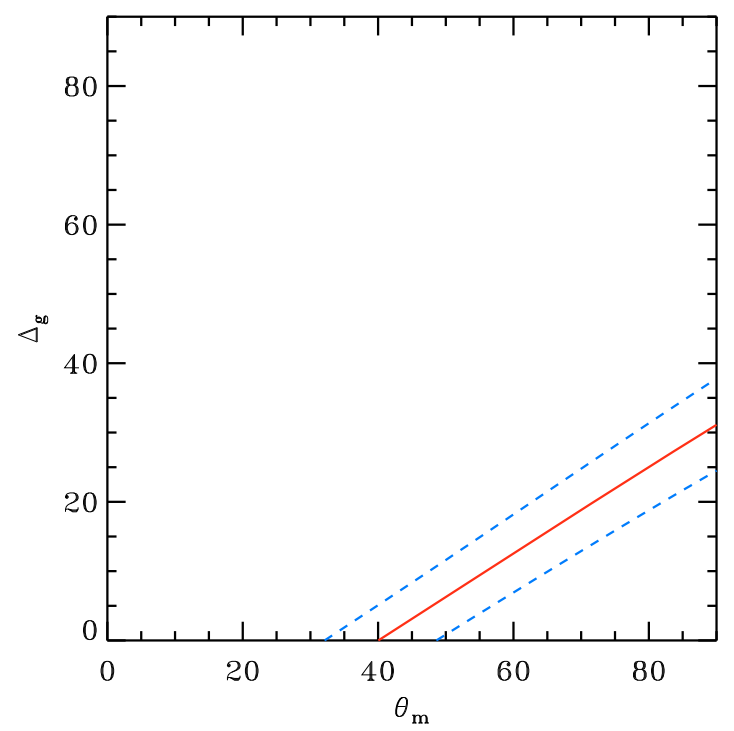

Fig. 9. Constraints on the maximum magnetic inclination $\theta_{\mathrm{m}}$ and maximum deflection angle $\Delta_{\mathrm{g}}$ for the blackbody pattern and full detectability of the secondary pulse. The solid line gives the most probable values and the dashed lines bound the $95 \%$ confidence region.

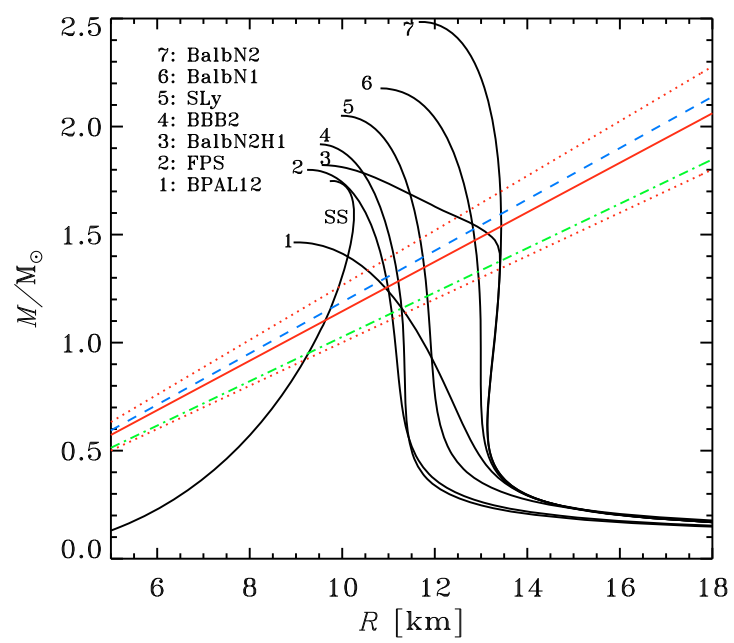

Fig. 10. Different mass-radius relations of neutron and strange stars equation of states (curves 1-7 and SS, see Suleimanov \& Poutanen 2006) and the obtained constraints on the compactness assuming a blackbody emission pattern and unconstrained magnetic inclination. The most probable value for the compactness $\left(\Delta_{\mathrm{g}}=31^{\circ}\right)$ is shown by the solid red line and the $95 \%$ confidence region is bound by red dotted lines. The dot-dashed-line shows the most probable value for the compactness (corresponding to $\Delta_{\mathrm{g}}=26^{\circ}$ ) obtained for the detection threshold $T=0.2$. The blue dashed line corresponds to the upper limits on mass as a function of radius obtained by B03.

Figure 10 shows various neutron star mass-radius relations and our best constraint corresponding to $\Delta_{\mathrm{g}}=31^{\circ}$.

Let us now look at the effect of the detection threshold which reduces the probability to observe double-peaked profiles (see Fig. 8a). For unconstrained magnetic inclination, we evaluate the probability to see double-peaked profiles by numerically computing the area on the $\cos i-\cos \theta$ plane where inequality (38) is satisfied. Compared to the case $T=0$, the maximum bending angle for $T=0.2$ decreases by just a few degrees to $\Delta_{\mathrm{g}}=26^{\circ} \pm 3^{\circ}$, which (for fixed $M$ ) would favor even larger neutron star radii (see Fig. 10). 
A similar study was made by B03, who obtained a different result for a number of reasons. Although our classification of X-ray pulsar light curves differs from B03 (we have 60 doublepeaked profiles out of 124, while B03 have 38/88), the probabilities $p_{0}$ are similar within a few percent. However, B03 assumed that single-peaked light curves would be observed only if one magnetic pole is seen (Class I). This led to the conclusion that the probability of having single-peaked profiles is about $21 \%$ for $\Delta_{\mathrm{g}}=0$ and this value decreases as compactness grows. Therefore in their point of view there is a conflict between the theory and observations, because the percentage of observed single-peaked light curves is much higher. As a result, B03 needed to constrain the magnetic field geometry (by introducing maximum magnetic inclination) in order to attain consistency between the observations and the theory. In our view, Class III is the only place to obtain double-peaked light curves. Therefore, we get $\theta_{\mathrm{m}} \gtrsim 35^{\circ}$ (and for reasonable value of the compactness the magnetic dipole inclination is random), while B03 got $\theta_{\mathrm{m}}<50^{\circ}$ (compare our Fig. 9 to Fig. 7 in B03).

\subsection{Modified pencil-beams and implications for neutron star compactness and magnetic field geometry}

The pulsars do not shine as blackbodies and therefore we now take a look at the effect of changing the emission pattern. For the $\cos ^{2} \alpha$ beam pattern, the probability of having double-peaked profiles is given by Eq. (33). If magnetic inclination is not constrained, $P_{\mathrm{d}}=0.79$, which significantly exceeds the observed fraction. Varying $\theta_{\mathrm{m}}$, we can get an agreement between the observations and the model. Requiring $P_{\mathrm{d}}=p_{0}$ (see Fig. 6), we get $\theta_{\mathrm{m}}=40^{\circ} \pm 4^{\circ}$, while if we account only for pulsars observed at energies above $10 \mathrm{keV}$ and take $P_{\mathrm{d}}=p_{1}$, then $\theta_{\mathrm{m}}=51^{\circ} \pm 4^{\circ}$. We note here that the probability to observe double-peaked profiles (33) does not depend on the deflection angle $\Delta_{\mathrm{g}}$ and thus we cannot get any constraints on the compactness of the neutron star (for a full detectability $T=0$ ). Other pencil beam models, $\cos ^{n} \alpha$ and $\cos \alpha(1+h \cos \alpha)$, give identical results. Thus even if the parameters describing the beam patterns of various NS have a large spread, the results are not affected.

The detection threshold may play an important role in classifying the pulse profiles. The expectation value for the fraction of the double-peaked profiles becomes lower and makes it rather close to the observed one for $T=0.2$, even if deviations from the blackbody pattern are not very large (see Fig. 8). Taking $n=2$, the predicted fraction of double-peaked profiles is about 0.57 , which is within $3 \sigma$ of the observed value $p_{0}=0.44$ and within $1 \sigma$ of the value $p_{1}=0.55$ obtained for pulsars observed above $10 \mathrm{keV}$ (see Table 1). Increasing $n$ makes the agreement even better. Again we stress that the predicted fraction depends only weakly on the neutron star compactness, which therefore cannot be constrained. Another conclusion is that the magnetic dipole inclination can be random. These results are not affected by our assumptions about the emission pattern if it is sufficiently far from the blackbody, because indices $n$ in the interval between about 1.5 and 6 and the anisotropy parameter $h>0.5$ predict similar fractions of double-peaked profiles (see Fig. 8).

The observed light curves are not always symmetric indicating the secondary pole is not at the antipodal position. To check how our assumption on antipodal positions of the spots affect the results, we have simulated the pulse profiles for two randomly positioned spots on the neutron star surface radiating as blackbodies. The probability to observe multiple-peaked light curves depends on the compactness of the neutron star reaching the maximum of $37 \%$ in the Newtonian limit (see the dasheddotted curve in Fig. 8a). This is much less than what is observed (between $44 \%$ and $55 \%$ ). A significant predicted fraction of multiple-peaked profiles (with number of peaks three or larger) even for the blackbody pattern contradicts the fact that such profiles are not observed. Other, more beamed patterns will just increase the fraction of multiple-peaked profiles. Thus even though the observed light curves indicate slight asymmetry, the simulations show that the spot positions cannot be completely random. This is consistent with the detailed models of the pulse profiles in several pulsars that show less than $10^{\circ}$ displacement of the dipole (Leahy \& Li 1995; Kraus et al. 1996). These small displacements will not affect our conclusions.

\section{Conclusions}

1. We have collected pulse profiles of $124 \mathrm{X}$-ray pulsars and magnetars and classified them according to the number of pulses visible in one rotational period. At energies above $10 \mathrm{keV}$, where the effects of photoelectric absorption and cyclotron line are minimal, $55 \%$ of the pulsars have doublepeaked profiles, while for all pulsars this fraction is $44 \%$.

2. We considered a simple model with two point-like antipodal spots emitting radiation according to different types of pencil-beam patterns $\cos ^{n} \alpha$ (where $\left.n \geq 1\right)$ and $\cos \alpha(1+$ $h \cos \alpha$ ) (with $h \geq 0$ ). The light curves produced by these cases are either single- or double-peaked depending on the model parameters.

3. We obtained some constraints on the radiation model parameters in the blackbody case, $n=1(h=0)$. The relative fraction of double-peaked profiles here depends on the neutron star compactness. The most probable values for the maximal gravitational light deflection angle is $\Delta_{\mathrm{g}} \sim 31^{\circ}$ (for unconstrained magnetic field, $\theta_{\mathrm{m}}=\pi / 2$ and with a full detectability of the secondary pulse). We also obtained a lower limit on the maximum magnetic inclination to be $\theta_{\mathrm{m}} \gtrsim 35^{\circ}$, and for reasonable NS compactnesses we have $\theta_{\mathrm{m}} \gtrsim 70^{\circ}$. When we include the effect of the detection threshold, the limit on the compactness is reduced to $\Delta_{\mathrm{g}} \lesssim 30^{\circ}$ (at $95 \%$ confidence) for $T=0.2$.

4. Any pencil-beam pattern (if not a blackbody) predicts a fixed fraction of double-peaked profiles of $79 \%$, which is inconsistent with the data. Restricting the maximum magnetic inclination reduces the fraction of double-peaked profiles. Comparison to the data gives us the most probable value for the maximum magnetic inclination of $\theta_{\mathrm{m}}=40^{\circ} \pm 4^{\circ}$. The neutron star compactness, however, cannot be constrained at all.

5. A limited detection sensitivity to weak pulses also reduces the fraction of double-peaked profiles. We found that this fraction depends weakly on the neutron star compactness and is consistent with the data for a large range of pencil-beam patterns at $T \sim 0.2$. In this case, we do not find good evidence that the magnetic inclination has a strict upper limit.

6. The overall conclusion is that contrary to the previous claims made by B03, the statistical method based on the classification of pulsar profiles by number of peaks cannot constrain the compactness of the neutron star. We also do not find univocal evidences in favor of the alignment of the magnetic dipole. It seems that the detailed analysis of the pulse profiles of individual pulsars and their evolution is the only way 
to obtain any useful constraints on the neutron star compactness and the magnetic field geometry.

Acknowledgements. This research was supported by Space institute, University of Oulu, and the Väisäla foundation (AM). J.P. acknowledges support from the Academy of Finland grant 110792. We also acknowledge the support of the International Space Science Institute (Bern, Switzerland), where part of this investigation was carried out. We thank Alexander Lutovinov and the referee for the valuable comments.

\section{References}

Angelini, L., Church, M. J., Parmar, A. N., Balucinska-Church, M., \& Mineo, T. 1998, A\&A, 339, L41

Aoki, T., Dotani, T., Ebisawa, K., et al. 1992, PASJ, 44, 641

Arons, J., \& Lea, S. M. 1980, ApJ, 235, 1016

Augello, G., Iaria, R., Robba, N. R., et al. 2003, ApJ, 596, L63

Bamba, A., Yokogawa, J., Ueno, M., Koyama, K., \& Yamauchi, S. 2001, PASJ, 53, 1179

Basko, M. M., \& Sunyaev, R. A. 1976, MNRAS, 175, 395

Becker, P. A., \& Wolff, M. T. 2005, ApJ, 621, L45

Beloborodov, A. M. 2002, ApJ, 566, L85

Beloborodov, A. M., \& Thompson, C. 2007, ApJ, 657, 967

Bildsten, L., Chakrabarty, D., Chiu, J., et al. 1997, ApJS, 113, 367

Bodaghee, A., Walter, R., Zurita Heras, J. A., et al. 2006, A\&A, 447, 1027

Bulik, T., Gondek-Rosińska, D., Santangelo, A., et al. 2003, A\&A, 404, 1023 (B03)

Bulik, T., Riffert, H., Meszaros, P., et al. 1995, ApJ, 444, 405

Burderi, L., di Salvo, T., Robba, N. R., et al. 1998, ApJ, 498, 831

Burderi, L., Di Salvo, T., Robba, N. R., La Barbera, A., \& Guainazzi, M. 2000, ApJ, 530, 429

Burnard, D. J., Arons, J., \& Klein, R. I. 1991, ApJ, 367, 575

Chakrabarty, D., Koh, T., Bildsten, L., et al. 1995, ApJ, 446, 826

Chernyakova, M., Lutovinov, A., Rodríguez, J., \& Revnivtsev, M. 2005 , MNRAS, 364, 455

Coe, M. J., Roche, P., Everall, C., et al. 1994, A\&A, 289, 784

Corbet, R. H. D., \& Peele, A. G. 1997, ApJ, 489, L83

Corbet, R. H. D., Marshall, F. E., Peele, A. G., \& Takeshima, T. 1999, ApJ, 517, 956

Corbet, R. H. D., Marshall, F. E., Coe, M. J., Laycock, S., \& Handler, G. 2001, ApJ, 548, L41

Cusumano, G., Israel, G. L., Mannucci, F., et al. 1998, A\&A, 337, 772

Cusumano, G., Maccarone, M. C., Nicastro, L., Sacco, B., \& Kaaret, P. 2000, ApJ, 528, L25

Davidson, K., \& Ostriker, J. P. 1973, ApJ, 179, 585

Edge, W. R. T., Coe, M. J., Galache, J. L., et al. 2004, MNRAS, 353, 1286

Finger, M. H., Bildsten, L., Chakrabarty, D., et al. 1999, ApJ, 517, 449

Finger, M. H., Macomb, D. J., Lamb, R. C., et al. 2001, ApJ, 560, 378

Galloway, D. K., Wang, Z., \& Morgan, E. H. 2005, ApJ, 635, 1217

Giacconi, R., Gursky, H., Kellogg, E., Schreier, E., \& Tananbaum, H. 1971, ApJ, 167, L67

Göğüş, E., Kouveliotou, C., Woods, P. M., Finger, M. H., \& van der Klis, M. 2002, ApJ, 577, 929

Haberl, F., \& Pietsch, W. 2005, A\&A, 438, 211

Haberl, F., \& Pietsch, W. 2008, A\&A, 484, 451

Haberl, F., Dennerl, K., Pietsch, W., \& Reinsch, K. 1997, A\&A, 318, 490

Haberl, F., Dennerl, K., \& Pietsch, W. 2003, A\&A, 406, 471

Haberl, F., Pietsch, W., Schartel, N., Rodriguez, P., \& Corbet, R. H. D. 2004a, A\&A, 420, L19

Haberl, F., Zavlin, V. E., Trümper, J., \& Burwitz, V. 2004b, A\&A, 419, 1077

Haensel, P., Potekhin, A. Y., \& Yakovlev, D. G. 2007, Neutron Stars 1: Equation of State and Structure, Astrophysics and Space Science Library, 326 (New York: Springer)

Hall, T. A., Finley, J. P., Corbet, R. H. D., \& Thomas, R. C. 2000, ApJ, 536, 450

Halpern, J. P., \& Gotthelf, E. V. 2005, ApJ, 618, 874

Halpern, J. P., \& Gotthelf, E. V. 2007, ApJ, 669, 579

Hulleman, F., in 't Zand, J. J. M., \& Heise, J. 1998, A\&A, 337, L25

Ibragimov, A., \& Poutanen, J. 2009, MNRAS, 400, 492

Imanishi, K., Yokogawa, J., Tsujimoto, M., \& Koyama, K. 1999, PASJ, 51, L15

in't Zand, J., \& Heise, J. 2004, The Astronomer's Telegram, 362, 1

in 't Zand, J. J. M., Baykal, A., \& Strohmayer, T. E. 1998, ApJ, 496, 386 in't Zand, J. J. M., Corbet, R. H. D., \& Marshall, F. E. 2001a, ApJ, 553, L165 in't Zand, J. J. M., Swank, J., Corbet, R. H. D., \& Markwardt, C. B. 2001b, A\&A, 380, L26

Israel, G. L., Stella, L., Angelini, L., et al. 1997a, ApJ, 484, L141
Israel, G. L., Stella, L., Angelini, L., et al. 1997b, ApJ, 474, L53 Israel, G. L., Campana, S., Covino, S., et al. 2000, ApJ, 531, L131 Israel, G. L., Campana, S., Dall'Osso, S., et al. 2007, ApJ, 664, 448 Iwasawa, K., Koyama, K., \& Halpern, J. P. 1992, PASJ, 44, 9 Karasev, D. I., Tsygankov, S. S., \& Lutovinov, A. A. 2008, MNRAS, 386, L10 Kelley, R. L., Rappaport, S., \& Ayasli, S. 1983, ApJ, 274, 765 Kinugasa, K., Torii, K., Hashimoto, Y., et al. 1998, ApJ, 495, 435 Kirk, J. G., Nagel, W., \& Storey, M. C. 1986, A\&A, 169, 259

Koh, D. T., Bildsten, L., Chakrabarty, D., et al. 1997, ApJ, 479, 933 Kohno, M., Yokogawa, J., \& Koyama, K. 2000, PASJ, 52, 299 Koyama, K., Kawada, M., Takeuchi, Y., et al. 1990, ApJ, 356, L47

Koyama, K., Kawada, M., Tawara, Y., et al. 1991a, ApJ, 366, L19 Koyama, K., Kunieda, H., Takeuchi, Y., \& Tawara, Y. 1991b, ApJ, 370, L77 Kraus, U. 2001, ApJ, 563, 289

Kraus, U., Blum, S., Schulte, J., Ruder, H., \& Meszaros, P. 1996, ApJ, 467, 794 Kraus, U., Zahn, C., Weth, C., \& Ruder, H. 2003, ApJ, 590, 424

Kuiper, L., Hermsen, W., den Hartog, P. R., \& Collmar, W. 2006, ApJ, 645, 556 Kuiper, L., Hermsen, W., \& Mendez, M. 2004, ApJ, 613, 1173

Lamb, R. C., Macomb, D. J., Prince, T. A., \& Majid, W. A. 2002, ApJ, 567, L129

Laycock, S., Corbet, R. H. D., Coe, M. J., et al. 2003, MNRAS, 339, 435

Laycock, S., Corbet, R. H. D., Perrodin, D., et al. 2002, A\&A, 385, 464

Laycock, S., Corbet, R. H. D., Coe, M. J., et al. 2005, ApJS, 161, 96

Leahy, D. A. 1990, MNRAS, 242, 188

Leahy, D. A. 2003, ApJ, 596, 1131

Leahy, D. A., \& Li, L. 1995, MNRAS, 277, 1177

Levine, A., Rappaport, S., Deeter, J. E., Boynton, P. E., \& Nagase, F. 1993, ApJ, 410,328

Levine, A. M., Rappaport, S., Remillard, R., \& Savcheva, A. 2004, ApJ, 617, 1284

Lutovinov, A., Revnivtsev, M., Gilfanov, M., et al. 2005a, A\&A, 444, 821

Lutovinov, A., Rodriguez, J., Revnivtsev, M., \& Shtykovskiy, P. 2005b, A\&A, 433, L41

Macomb, D. J., Finger, M. H., Harmon, B. A., Lamb, R. C., \& Prince, T. A. 1999, ApJ, 518, L99

Macomb, D. J., Fox, D. W., Lamb, R. C., \& Prince, T. A. 2003, ApJ, 584, L79

Majid, W. A., Lamb, R. C., \& Macomb, D. J. 2004, ApJ, 609, 133

McBride, V. A., Wilms, J., Coe, M. J., et al. 2006, A\&A, 451, 267

McClintock, J. E., Nugent, J. J., Li, F. K., \& Rappaport, S. A. 1977, ApJ, 216, L15

Mereghetti, S. 2008, A\&AR, 15, 225

Mereghetti, S., Tiengo, A., Israel, G. L., \& Stella, L. 2000, A\&A, 354, 567

Mereghetti, S., Chiarlone, L., Israel, G. L., \& Stella, L. 2002, in Neutron Stars,

Pulsars, and Supernova Remnants, ed. W. Becker, H. Lesch, \& J. Trümper, 29

Mereghetti, S., Götz, D., Mirabel, I. F., \& Hurley, K. 2005, A\&A, 433, L9

Meszaros, P., \& Nagel, W. 1985a, ApJ, 298, 147

Meszaros, P., \& Nagel, W. 1985b, ApJ, 299, 138

Mihara, T. 1995, Ph.D. Thesis, Dept. of Physics, Univ. of Tokyo

Molkov, S., Hurley, K., Sunyaev, R., et al. 2005, A\&A, 433, L13

Morii, M., Sato, R., Kataoka, J., \& Kawai, N. 2003, PASJ, 55, L45

Morris, D. C., Smith, R. K., Markwardt, C. B., et al. 2009, ApJ, 699, 892

Nagase, F. 1989, PASJ, 41, 1

Nagel, W. 1981a, ApJ, 251, 288

Nagel, W. 1981b, ApJ, 251, 278

Negueruela, I., Reig, P., \& Clark, J. S. 2000, A\&A, 354, L29

Oosterbroek, T., Orlandini, M., Parmar, A. N., et al. 1999, A\&A, 351, L33

Paul, B., \& Rao, A. R. 1998, A\&A, 337, 815

Pechenick, K. R., Ftaclas, C., \& Cohen, J. M. 1983, ApJ, 274, 846

Poutanen, J. 2008, in A Decade of Accreting Millisecond X-ray Pulsars, ed.

R. Wijnands, D. Altamirano, P. Soleri, N. Degenaar, N. Rea, P. Casella,

A. Patruno, \& M. Linares (New York: AIP), AIP Conf. Ser. 1068, 77

Poutanen, J., \& Beloborodov, A. M. 2006, MNRAS, 373, 836

Poutanen, J., \& Gierliński, M. 2003, MNRAS, 343, 1301

Reig, P., \& Roche, P. 1999a, MNRAS, 306, 100

Reig, P., \& Roche, P. 1999b, MNRAS, 306, 95

Reig, P., Belloni, T., Israel, G. L., et al. 2008, A\&A, 485, 797

Robba, N. R., \& Warwick, R. S. 1989, ApJ, 346, 469

Sakano, M., Torii, K., Koyama, K., Maeda, Y., \& Yamauchi, S. 2000, PASJ, 52, 1141

Santangelo, A., Cusumano, G., dal Fiume, D., et al. 1998, A\&A, 338, L59

Santangelo, A., Segreto, A., Giarrusso, S., et al. 1999, ApJ, 523, L85

Sasaki, M., Pietsch, W., \& Haberl, F. 2003, A\&A, 403, 901

Schmidtke, P. C., Cowley, A. P., McGrath, T. K., \& Anderson, A. L. 1995, PASP, 107,450

Scott, D. M., Finger, M. H., Wilson, R. B., et al. 1997, ApJ, 488, 831

Seward, F. D., Charles, P. A., \& Smale, A. P. 1986, ApJ, 305, 814

Sguera, V., Hill, A. B., Bird, A. J., et al. 2007, A\&A, 467, 249

Sidoli, L., Romano, P., Mereghetti, S., et al. 2007, A\&A, 476, 1307 
Skinner, G. K., Bedford, D. K., Elsner, R. F, et al. 1982, Nature, 297, 568 Stollberg, M. T., Finger, M. H., Wilson, R. B., et al. 1999, ApJ, 512, 313 Suleimanov, V., \& Poutanen, J. 2006, MNRAS, 369, 2036

Tawara, Y., Yamauchi, S., Awaki, H., et al. 1989, PASJ, 41, 473

Thompson, C., \& Beloborodov, A. M. 2005, ApJ, 634, 565

Thorsett, S. E., \& Chakrabarty, D. 1999, ApJ, 512, 288

Torii, K., Kinugasa, K., Katayama, K., et al. 1998a, ApJ, 508, 854

Torii, K., Kinugasa, K., Katayama, K., Tsunemi, H., \& Yamauchi, S. 1998b, ApJ, 503,843

Torii, K., Sugizaki, M., Kohmura, T., Endo, T., \& Nagase, F. 1999, ApJ, 523, L65

Trudolyubov, S. P. 2008, MNRAS, 387, L36

Trudolyubov, S., Kotov, O., Priedhorsky, W., Cordova, F., \& Mason, K. 2005, ApJ, 634, 314

Trudolyubov, S. P., Priedhorsky, W. C., \& Córdova, F. A. 2007, ApJ, 663, 487

Tsujimoto, M., Imanishi, K., Yokogawa, J., \& Koyama, K. 1999, PASJ, 51, L21

Tsygankov, S. S., \& Lutovinov, A. A. 2005, Astr. Lett., 31, 88

Tsygankov, S. S., Lutovinov, A. A., Churazov, E. M., \& Sunyaev, R. A. 2006, MNRAS, 371, 19
Ueno, M., Yokogawa, J., Imanishi, K., \& Koyama, K. 2000, PASJ, 52, L63

Viironen, K., \& Poutanen, J. 2004, A\&A, 426, 985

Wang, Y.-M., \& Welter, G. L. 1981, A\&A, 102, 97

Wilson, C. A., Finger, M. H., Gögüuss, E., Woods, P. M., \& Kouveliotou, C. 2002, ApJ, 565, 1150

Wilson, C. A., Finger, M. H., Coe, M. J., \& Negueruela, I. 2003, ApJ, 584, 996

Woo, J. W., Clark, G. W., Levine, A. M., Corbet, R. H. D., \& Nagase, F. 1996, ApJ, 467, 811

Yahel, R. Z. 1980, A\&A, 90, 26

Yokogawa, J., Imanishi, K., Tsujimoto, M., Kohno, M., \& Koyama, K. 1999, PASJ, 51, 547

Yokogawa, J., Imanishi, K., Tsujimoto, M., et al. 2000a, ApJS, 128, 491

Yokogawa, J., Imanishi, K., Ueno, M., \& Koyama, K. 2000b, PASJ, 52, L73

Yokogawa, J., Paul, B., Ozaki, M., et al. 2000c, ApJ, 539, 191

Yokogawa, J., Torii, K., Imanishi, K., \& Koyama, K. 2000d, PASJ, 52, L37

Yokogawa, J., Torii, K., Kohmura, T., Imanishi, K., \& Koyama, K. 2000e, PASJ, 52, L53

Yokogawa, J., Torii, K., Kohmura, T., \& Koyama, K. 2001, PASJ, 53, L9 